\title{
The curvature invariant for a class of homogeneous operators
}

\author{
Gadadhar Misra and Subrata Shyam Roy
}

\begin{abstract}
For an operator $T$ in the class $\mathrm{B}_{n}(\Omega)$, introduced by Cowen and Douglas, the simultaneous unitary equivalence class of the curvature and the covariant derivatives up to a certain order of the corresponding bundle $E_{T}$ determine the unitary equivalence class of the operator $T$. In a subsequent paper, the authors ask if the simultaneous unitary equivalence class of the curvature and these covariant derivatives are necessary to determine the unitary equivalence class of the operator $T \in \mathrm{B}_{n}(\Omega)$. Here we show that some of the covariant derivatives are necessary. Our examples consist of homogeneous operators in $\mathrm{B}_{n}(\mathbb{D})$. For homogeneous operators, the simultaneous unitary equivalence class of the curvature and all its covariant derivatives at any point $w$ in the unit disc $\mathbb{D}$ are determined from the simultaneous unitary equivalence class at 0 . This shows that it is enough to calculate all the invariants and compare them at just one point, say 0 . These calculations are then carried out in number of examples. One of our main results is that the curvature along with its covariant derivative of order $(0,1)$ at 0 determines the equivalence class of generic homogeneous Hermitian holomorphic vector bundles over the unit disc.
\end{abstract}

\section{Introduction}

Let $\mathcal{H}$ be a Hilbert space of holomorphic functions on a bounded open connected set $\Omega \subseteq \mathbb{C}$. Assume that for $w \in \Omega$, the point evaluations $\operatorname{ev}_{w}: f \mapsto f(w)$ on $\mathcal{H}$ are bounded and locally bounded. Then the Hilbert space $\mathcal{H}$ possesses a reproducing kernel (cf. [2]) $K$, that is,

$$
f(w)=\left\langle f, K_{w}\right\rangle, \quad f \in \mathcal{H}, \quad K_{w} \in \mathcal{H} \text { for all } w \in \Omega .
$$

The map $w \mapsto K_{\bar{w}}$ is holomorphic and defines a holomorphic vector bundle on $\Omega^{*}:=\{w: \bar{w} \in$ $\Omega\}$. It turns out that if the adjoint of the multiplication by the coordinate function on $\mathcal{H}$ is bounded then $K_{w}$ is an eigenvector for it with eigenvalue $\bar{w}, w \in \Omega$.

Suppose that $T$ is a bounded linear operator on a Hilbert space $\mathcal{H}$ possessing an open set of eigenvalues, say $\Omega$, with constant multiplicity 1 . For $w \in \Omega$, let $\gamma_{w}$ be the eigenvector for $T$ with eigenvalue $w$. In a significant paper [7], Cowen and Douglas showed that for these operators $T$, under some additional mild hypothesis, one may choose the eigenvector $\gamma_{w}$ to ensure that the map $w \mapsto \gamma_{w}$ is holomorphic. Thus the operator $T$ gives rise to a holomorphic Hermitian vector bundle $E_{T}$ on $\Omega$. They proved the following.

(i) The equivalence class of the holomorphic Hermitian bundle $E_{T}$ determines the unitary equivalence class of the operator $T$.

(ii) The operator $T$ is unitarily equivalent to the adjoint of the multiplication by the coordinate function on a Hilbert space $\mathcal{H}$ of holomorphic functions on $\Omega^{*}$. The point evaluation on $\mathcal{H}$ is shown to be bounded and locally bounded assuring the existence of a reproducing kernel function for $\mathcal{H}$.

Received 22 June 2007; published online 25 March 2009.

2000 Mathematics Subject Classification 47B32 (primary), 46E22, 22D10, 22F50 (secondary).

The research of the first author was supported in part by a grant from the DST-NSF Science and Technology Cooperation Programme. The second author was supported by the Indian Statistical Institute. 
From (i), as shown in [7], it follows that the curvature

$$
\mathcal{K}(w):=\frac{\partial}{\partial w} \frac{\partial}{\partial \bar{w}} \log \left\|\gamma_{w}\right\|^{2}, w \in \Omega
$$

of the line bundle $E_{T}$ is a complete invariant for the operator $T$. On the other hand, following (ii), Curto and Salinas [10] showed that the normalized kernel

$$
\tilde{K}(z, w)=K\left(w_{0}, w_{0}\right)^{1 / 2} K\left(z, w_{0}\right)^{-1} K(z, w) K\left(w_{0}, w\right)^{-1} K\left(w_{0}, w_{0}\right)^{1 / 2}, \quad w \in \Omega
$$

at $w_{0} \in \Omega$ is a complete invariant for the operator $T$ as well.

If the dimension of the eigenspace of the operator $T$ at $w$ is no longer assumed to be 1 , then a complete set of unitary invariants for the operator $T$ involves not only the curvature but also a certain number of its covariant derivatives. The reproducing kernel, in this case, takes values in the $n \times n$ matrices $\mathcal{M}_{n}$, where $n$ is the (constant) dimension of the eigenspace of the operator $T$ at $w$. The normalized kernel, modulo conjugation by a fixed unitary matrix from $\mathcal{M}_{n}$, continues to provide a complete invariant for the operator $T$.

Unfortunately, very often, the computation of these invariants tends to be hard. However, there is one situation, where these computations become somewhat tractable, namely, if $T$ is assumed to be homogeneous. Recall that an operator $T$ is said to be homogeneous if $\varphi(T)$ is unitarily equivalent to $T$ for all $\varphi$ in the bi-holomorphic automorphism group Möb of the unit disc $\mathbb{D}$. For a homogeneous operator $T$, if the unitary operator $U_{\varphi}$ implementing the unitary equivalence between $T$ and $\varphi(T)$ can be chosen such that the map $\varphi \mapsto U_{\varphi}$ is a projective unitary representation of the group Möb, then $U_{\varphi}$ is said to be the representation associated to $T$. If the operator $T$ is assumed to be irreducible, then the existence of an associated representation is easily established using the Schur lemma (cf. [5, Theorem 2.2]).

One may expect that in the case of homogeneous operators, the form of the invariants, discussed above, at any one point will determine it completely. We illustrate this phenomenon throughout the paper. Homogeneous operators have been studied extensively over the last few years $[1,3-6,14,15,17,19]$. Some of these homogeneous operators correspond to a holomorphic Hermitian homogeneous bundle, as discussed above. Recall that a Hermitian holomorphic bundle $E$ on the open unit disc $\mathbb{D}$ is homogeneous if every $\varphi$ in Möb lifts to an isometric bundle map of $E$.

Although, the homogeneous bundles $E$ on the open unit disc $\mathbb{D}$ have been classified in $[\mathbf{6}]$, it is not easy to determine which of these homogeneous bundles $E$ comes from a homogeneous operators. In [19], Wilkins used his classification to describe all the irreducible homogeneous operators of rank 2. In the paper [15], the first author along with Koranyi, gives an explicit description of a class of homogeneous bundles and the corresponding homogeneous operator, thus making it possible for us to compute the curvature invariants for these homogeneous operators. Although, our main focus will be on the computation of the curvature invariants, we will also compute the normalized kernel and explain the relationship between these two types of invariants. Along the way, we give a partial answer to some questions raised in $[\mathbf{7}, \mathbf{9}]$.

For a bounded open connected set $\Omega \subseteq \mathbb{C}$ and $n \in \mathbb{N}$, let us recall that the class $\mathrm{B}_{n}(\Omega)$, introduced in [7], consists of bounded operators $T$ with the following properties:

(a) $\Omega \subset \sigma(T)$;

(b) $\operatorname{ran}(T-w)=\mathcal{H}$ for $w \in \Omega$;

(c) $\bigvee_{w \in \Omega} \operatorname{ker}(T-w)=\mathcal{H}$ for $w \in \Omega$;

(d) $\operatorname{dim} \operatorname{ker}(T-w)=n$ for $w \in \Omega$.

It was shown in [7, Proposition 1.11] that the eigenspaces for each $T$ in $\mathrm{B}_{n}(\Omega)$ form a Hermitian holomorphic vector bundle $E_{T}$ over $\Omega$, that is,

$$
E_{T}:=\{(w, x) \in \Omega \times \mathcal{H}: x \in \operatorname{ker}(T-w)\}, \quad \pi(w, x)=w
$$


and there exists a holomorphic frame $w \mapsto \gamma(w):=\left(\gamma_{1}(w), \ldots, \gamma_{n}(w)\right)$ with $\gamma_{i}(w) \in \operatorname{ker}(T-$ $w), 1 \leqslant i \leqslant n$. The Hermitian structure at $w$ is the one that $\operatorname{ker}(T-w)$ inherits as a subspace of the Hilbert space $\mathcal{H}$. In other words, the metric at $w$ is simply the grammian $h(w)=\left(\left(\left\langle\gamma_{j}(w), \gamma_{i}(w)\right\rangle\right)\right)_{i, j=1}^{n}$. The curvature $\mathcal{K}_{T}(w)$ of the bundle $E_{T}$ is then defined to be $\frac{\partial}{\partial \bar{w}}\left(h^{-1} \frac{\partial}{\partial w} h\right)(w)$ for $w \in \Omega$ (cf. [18, pp. 78, 79]).

TheOREM 1.1 ([8, p. 326]). Two operators $T, \tilde{T}$ in $\mathrm{B}_{1}(\Omega)$ are unitarily equivalent if and only if $\mathcal{K}_{T}(w)=\mathcal{K}_{\widetilde{T}}(w)$ for $w$ in $\Omega$.

Thus the curvature of the line bundle $E_{T}$ is a complete set of unitary invariant for an operator in $\mathrm{B}_{1}(\Omega)$. Although, more complicated, a complete set of unitary invariants for the operators in the class $\mathrm{B}_{n}(\Omega)$ is given in [7].

It is not hard to see (cf. [18, p. 72]) that the curvature of a bundle $E$ transforms according to the rule $\mathcal{K}(f g)(w)=\left(g^{-1} \mathcal{K}(f) g\right)(w), w \in \Delta$, where $f=\left(e_{1}, \ldots, e_{n}\right)$ is a frame for $E$ over an open subset $\Delta \subseteq \Omega$ and $g: \Delta \rightarrow G L(n, \mathbb{C})$ is a holomorphic change of frame. For a line bundle $E$, locally, the change of frame $g$ is a scalar-valued holomorphic function. In this case, it follows from the transformation rule for the curvature that it is independent of the choice of a frame. In general, the curvature of a bundle $E$ of rank $n>1$ depends on the choice of a frame. Thus the curvature $\mathcal{K}$ itself cannot be an invariant for the bundle $E$. However, the eigenvalues of $\mathcal{K}$ are invariants for the bundle $E$. More interesting is the description of a complete set of invariants given in [7, Definition 2.17 and Theorem 3.17] involving the curvature and the covariant derivatives

$$
\mathcal{K}_{z^{i} \bar{z}^{j}}, \quad 0 \leqslant i \leqslant j \leqslant i+j \leqslant n, \quad(i, j) \neq(0, n),
$$

where rank of $E=n$. In a subsequent paper (cf. [9, p. 78]), by means of examples, Cowen and Douglas showed that fewer covariant derivatives of the curvature will not suffice to determine the class of the bundle $E$. These examples do not necessarily correspond to an operator in the class $\mathrm{B}_{n}(\Omega)$. Recall that if a Hermitian holomorphic vector bundle $E$ is the pull-back of the tautological bundle defined over the Grassmannian $\mathcal{G} r(n, \mathcal{H})$ under the holomorphic map

$$
t: \Omega \longrightarrow \mathcal{G} r(n, \mathcal{H}), \quad t(w)=\operatorname{ker}(T-w), w \in \Omega
$$

for some operator $T: \mathcal{H} \rightarrow \mathcal{H}, T \in \mathrm{B}_{n}(\Omega)$, then $E=E_{T}$ and we say that it corresponds to the operator $T$. On the other hand, for certain class of operators like the generalized Wilkins operators $\mathcal{W}_{k}:=\left\{M_{k}^{(\alpha, \beta)}: \alpha, \beta>0\right\} \subseteq \mathrm{B}_{k+1}(\mathbb{D})$ described below (cf. [4, p. 428]), the unitary equivalence class of the curvature $\mathcal{K}$ (just at one point) determines the unitary equivalence class of these operators in $\mathcal{W}_{k}$. This is easily proved using the form of the curvature at 0 of the generalized Wilkins operators $M_{k}^{(\alpha, \beta)}$, namely, $\operatorname{diag}(\alpha, \ldots, \alpha, \alpha+(k+1) \beta+$ $k(k+1))$ (cf. [16, Theorem 4.12]).

In this paper we construct examples of operators $T$ in $\mathrm{B}_{2}(\mathbb{D})$ and $\mathrm{B}_{3}(\mathbb{D})$ to show that the eigenvalues of the curvature for the corresponding bundle $E_{T}$ do not necessarily determine the class of the bundle $E_{T}$. Our examples consisting of homogeneous bundles $E_{T}$ show that the covariant derivatives of the curvature up to order $(1,1)$ cannot be dropped, in general, from the set of invariants described above. These verifications are somewhat non-trivial and use the homogeneity of the bundle in an essential way. It is not clear if for a homogeneous bundle the curvature along with its derivatives up to order $(1,1)$ suffices to determine its equivalence class. Secondly, the original question of sharpness of $[\mathbf{7}$, p. 214] and [9, p. 39] remains open, although our examples provide a partial answer.

One of the main theorems we prove here involves the class of operators constructed in [15]. This construction provides a complete list (up to mutual unitary inequivalence) of irreducible homogeneous operators in $\mathrm{B}_{k+1}(\mathbb{D}), k \geqslant 1$, whose associated representation is multiplicity-free. 
It turns out that for $k=1$, this is exactly the same list as that of Wilkins [19], namely, $\mathcal{W}_{1}$. However, for $k \geqslant 2$, the class of operators $\mathcal{W}_{k} \subseteq \mathrm{B}_{k+1}(\mathbb{D})$ is much smaller than the corresponding list from [15]. Now consider those homogeneous and irreducible operators from [15] for which the eigenvalues of the curvature are distinct and have multiplicity 1 . The Hermitian holomorphic bundles corresponding to such operators are called generic (cf. [7, p. 226]). We show that for these operators, the simultaneous unitary equivalence class of the curvature and the covariant derivative of order $(0,1)$ at 0 determine the unitary equivalence class of the operator $T$. This is considerably more involved than the corresponding result for the class $\mathcal{W}_{k}$.

To summarize, it is surprising that there are no known examples of operators $T \in \mathrm{B}_{n}(\Omega)$, $n>1$, for which the set of eigenvalues of the curvature $\mathcal{K}_{T}$ is not a complete invariant. We construct some examples in this paper to show that one needs the covariant derivatives of the curvature as well to determine the unitary equivalence class of an operator $T \in \mathrm{B}_{n}(\Omega), n>1$. The inherent difficulty in finding such examples suggests the possibility that the complete set of invariants for an operator $T \in \mathrm{B}_{n}(\Omega)$ described in $[\mathbf{7}, \mathbf{9}]$ may not be the most economical. Although, in these papers, it is shown that for generic bundles, the set of complete invariants is much smaller and consists of the curvature and its covariant derivatives of order $(0,1)$ and $(1,1)$. However, even for generic bundles, it is not clear if this is the best possible. Indeed, we show that for a certain class of homogeneous operators corresponding to generic holomorphic Hermitian homogeneous bundles, the curvature along with its covariant derivative of order $(0,1)$ at 0 provides a complete set of invariants.

\section{Examples from the jet construction}

Let $B(z, w)=(1-z \bar{w})^{-2}$ be the Bergman kernel on the unit disc, and let the Hilbert space corresponding to the non-negative definite kernel $B^{\lambda / 2}(z, w)=(1-z \bar{w})^{-\lambda}$ be $\mathbb{A}^{(\lambda)}(\mathbb{D})$ for $\lambda>0$. We let $M^{(\lambda)}: \mathbb{A}^{(\lambda)}(\mathbb{D}) \rightarrow \mathbb{A}^{(\lambda)}(\mathbb{D})$ be the multiplication operator, that is, $\left(M^{(\lambda)} f\right)(z)=$ $z f(z), f \in \mathbb{A}^{(\lambda)}(\mathbb{D}), z \in \mathbb{D}$. Following the jet construction of $[\mathbf{1 2}]$ (see also $[\mathbf{1 1}, \mathbf{1 3}, \mathbf{1 7}]$ ), we construct a Hilbert space $A_{k}^{(\alpha, \beta)}(\mathbb{D})(\alpha, \beta>0, k \in \mathbb{N})$ starting from the kernel Hilbert space $\mathbb{A}^{(\alpha)}(\mathbb{D}) \otimes \mathbb{A}^{(\beta)}(\mathbb{D})$ with reproducing kernel $B^{(\alpha, \beta)}(\mathbf{z}, \mathbf{w})=B^{\alpha / 2}\left(z_{1}, w_{1}\right) B^{\beta / 2}\left(z_{2}, w_{2}\right), \mathbf{z}=$ $\left(z_{1}, z_{2}\right), \mathbf{w}=\left(w_{1}, w_{2}\right) \in \mathbb{D}^{2}$. The Hilbert space $A_{k}^{(\alpha, \beta)}(\mathbb{D})$ consists of $\mathbb{C}^{k+1}$-valued holomorphic functions defined on the open unit disc $\mathbb{D}$. It turns out that the reproducing kernel $B_{k}^{(\alpha, \beta)}$ for $A_{k}^{(\alpha, \beta)}(\mathbb{D})$ is

$$
B_{k}^{(\alpha, \beta)}(z, w)=\left(\left(B^{\alpha / 2}\left(z_{1}, w_{1}\right) \partial_{z_{2}}^{i} \partial_{\bar{w}_{2}}^{j} B^{\beta / 2}\left(z_{2}, w_{2}\right)\right)\right)_{0 \leqslant i, j \leqslant k \mid \text { res } \mathbb{D} \times \mathbb{D}},
$$

that is, $z_{1}=z=z_{2}$ and $w_{1}=w=w_{2}$. The multiplication operator on $A_{k}^{(\alpha, \beta)}(\mathbb{D})$ is denoted by $M_{k}^{(\alpha, \beta)}$.

ExAmple 2.1. Consider the operators $M:=M^{(\lambda)} \oplus M^{(\mu)}$ and $M^{\prime}:=M_{1}^{(\alpha, \beta)}$ for $\lambda, \mu, \alpha, \beta>0$. Wilkins $[\mathbf{1 9}]$ has shown that the operator $M^{\prime *}$ is in $\mathrm{B}_{2}(\mathbb{D})$ and that it is irreducible. This operator is also homogeneous; that is, $\varphi\left(M^{\prime}\right)$ is unitarily equivalent to $M^{\prime}$ for all bi-holomorphic automorphisms $\varphi$ of the open unit disc $\mathbb{D}$ (cf. [4]). It is easy to see that the operators $M^{(\lambda)}$ and $M^{(\mu)}$ are both homogeneous and the adjoint of these operators is in the class $\mathrm{B}_{1}(\mathbb{D})$. Consequently, the direct sum, namely, $M^{*}$ is homogeneous and lies in the class $\mathrm{B}_{2}(\mathbb{D})$. Let

(1) $h(z)=\left(\begin{array}{cc}B^{\lambda / 2}(z, z) & 0 \\ 0 & B^{\mu / 2}(z, z)\end{array}\right), \lambda, \mu>0$,

(2) $h^{\prime}(z)=B_{1}^{(\alpha, \beta)}(z, z)^{\operatorname{tr}}=\left(\begin{array}{cc}\left(1-|z|^{2}\right)^{2} & \beta \bar{z}\left(1-|z|^{2}\right) \\ \beta z\left(1-|z|^{2}\right) & \beta\left(1+\beta|z|^{2}\right)\end{array}\right)\left(1-|z|^{2}\right)^{-\alpha-\beta-2}, \alpha, \beta>0$, for $z \in \mathbb{D}$, where $X^{\text {tr }}$ denotes the transpose of the matrix $X$. 
The bundles $(E, h)$ and $\left(E^{\prime}, h^{\prime}\right)$ correspond to the operators $M^{*}$ and $M^{\prime *}$, respectively. We denote the curvature and the covariant derivative of the curvature of order $(0,1)$ for the bundles $(E, h)$ and $\left(E^{\prime}, h^{\prime}\right)$ by $\mathcal{K}, \mathcal{K}_{\bar{z}}$ and $\mathcal{K}^{\prime}, \mathcal{K}_{\bar{z}}^{\prime}$, respectively. By direct computation we have

$$
\begin{aligned}
\mathcal{K}(z) & =\left(\begin{array}{cc}
\frac{\lambda}{\left(1-|z|^{2}\right)^{2}} & 0 \\
0 & \frac{\mu}{\left(1-|z|^{2}\right)^{2}}
\end{array}\right), \quad \mathcal{K}_{\bar{z}}(z)=2\left(\begin{array}{cc}
\frac{\lambda z}{\left(1-|z|^{2}\right)^{3}} & 0 \\
0 & \frac{\mu z}{\left(1-|z|^{2}\right)^{3}}
\end{array}\right) ; \\
\mathcal{K}^{\prime}(z) & =\left(\begin{array}{cc}
\frac{\alpha}{\left(1-|z|^{2}\right)^{2}} & \frac{-2 \beta(\beta+1) \bar{z}}{\left(1-|z|^{2}\right)^{3}} \\
0 & \frac{\alpha+2 \beta+2}{\left(1-|z|^{2}\right)^{2}}
\end{array}\right), \quad \mathcal{K}_{\bar{z}}^{\prime}(z)=2\left(\begin{array}{cc}
\frac{\alpha z}{\left(1-|z|^{2}\right)^{3}} & \frac{-\beta(\beta+1)\left(1+2|z|^{2}\right)}{\left(1-|z|^{2}\right)^{4}} \\
0 & \frac{(\alpha+2 \beta+2) z}{\left(1-|z|^{2}\right)^{3}}
\end{array}\right) .
\end{aligned}
$$

Choose $\lambda, \mu>0$ with $\mu-\lambda>2$ and set $\alpha=\lambda$ and $\beta=\frac{1}{2}(\mu-\lambda-2)$. Since curvature is selfadjoint, the set of eigenvalues is a complete set of unitary invariants for the curvature. The eigenvalues for $\mathcal{K}(z)$ and $\mathcal{K}^{\prime}(z), z \in \mathbb{D}$, are clearly the same by the choice of $\lambda, \mu, \alpha$ and $\beta$. Therefore, these matrices are pointwise unitarily equivalent. Now, we observe that $\mathcal{K}_{\bar{z}}(0)=0$ and $\mathcal{K}_{\bar{z}}^{\prime}(0) \neq 0$. Hence they cannot be unitarily equivalent. It follows that the eigenvalues of the curvature alone cannot determine the unitary equivalence class of the bundle. However, in this example, the covariant derivative of order $(0,1)$ suffices to distinguish the equivalence class of the operators $M$ and $M^{\prime *}$.

Before we construct the next example, let us recall that for any reproducing kernel $K$ on $\mathbb{D}$, the normalized kernel $\tilde{K}(z, w)$ at 0 (in the sense of Curto-Salinas [10, Remark 4.7(b)]) is defined to be the kernel $K(0,0)^{1 / 2} K(z, 0)^{-1} K(z, w) K(0, w)^{-1} K(0,0)^{1 / 2}$. This kernel is characterized by the property $\tilde{K}(z, 0)=I$ for $z \in \mathbb{D}$ and is therefore uniquely determined up to a conjugation by a constant unitary matrix. Let $K(z, w)=\sum_{k, \ell \geqslant 0} a_{k \ell} z^{k} \bar{w}^{\ell}$ and $\tilde{K}(z, w)=\sum_{k, \ell \geqslant 0} \tilde{a}_{k \ell} z^{k} \bar{w}^{\ell}$, where $a_{k \ell}$ and $\tilde{a}_{k \ell}$ are determined by the real analytic functions $K$ and $\tilde{K}$, respectively, $a_{k \ell}$ and $\tilde{a}_{k \ell}$ are in $\mathcal{M}_{n}$, for $k, \ell \geqslant 0$. Since $\tilde{K}(z, w)$ is a normalized kernel, it follows that $\tilde{a}_{00}=$ $I$ and $\tilde{a}_{k 0}=\tilde{a}_{0 \ell}=0$ for $k, \ell \geqslant 1$. Let $K(z, w)^{-1}=\sum_{k, \ell \geqslant 0} b_{k \ell} z^{k} \bar{w}^{\ell}$, where $b_{k \ell}$ is in $\mathcal{M}_{n}$ for $k, \ell \geqslant 0$. Clearly, $K(z, w)^{*}=K(w, z)$ for any reproducing kernel $K$ and $z, w \in \mathbb{D}$. Therefore, $a_{k \ell}^{*}=a_{\ell k}, \tilde{a}_{k \ell}^{*}=\tilde{a}_{\ell k}$ and $b_{k \ell}{ }^{*}=b_{\ell k}$ for $k, \ell \geqslant 0$, where $X^{*}$ denotes the conjugate transpose of the matrix $X$.

Let $\mathcal{H}$ be a Hilbert space of holomorphic functions on $\mathbb{D}$ possessing the reproducing kernel $K$. To emphasize the role of the reproducing kernel, we sometimes write $(\mathcal{H}, K)$ for this Hilbert space. If we assume that the adjoint $M^{*}$ of the multiplication operator $M$ on the Hilbert space $(\mathcal{H}, K)$ is in $\mathrm{B}_{k}(\mathbb{D})$, then it follows from $\left[\mathbf{1 0}\right.$, Lemma 4.8, p. 474] that the operator $\tilde{M}^{*}$ on the Hilbert space $\tilde{\mathcal{H}}$ determined by the normalized kernel $\tilde{K}$ is unitarily equivalent to $M^{*}$ on the Hilbert space $(\mathcal{H}, K)$. Hence the adjoint of the multiplication operator $\tilde{M}$ on $(\tilde{\mathcal{H}}, \tilde{K})$ lies in $\mathrm{B}_{k}(\mathbb{D})$ as well. Let $(\tilde{E}, \tilde{h})$ be the corresponding bundle, where $\tilde{h}(z)=\tilde{K}(z, z)^{\operatorname{tr}}, z \in \mathbb{D}$. The curvature of this bundle is $\tilde{\mathcal{K}}(z)=\frac{\partial}{\partial \bar{z}}\left(\tilde{h}^{-1} \frac{\partial}{\partial z} \tilde{h}\right)(z)$ for $z \in \mathbb{D}$.

Lemma 2.2. Let $\tilde{h}(z)^{\operatorname{tr}}=\tilde{K}(z, z)=\sum_{k, \ell \geqslant 0} a_{k \ell} z^{k} \bar{z}^{\ell}$. In this notation, we have

(a) $\partial^{m} \tilde{h}(0)=\bar{\partial}^{n} \tilde{h}(0)=0=\partial^{m} \tilde{h}^{-1}(0)=\bar{\partial}^{n} \tilde{h}^{-1}(0)$ for $m, n \geqslant 1$ and

(b) $\bar{\partial} \partial \tilde{h}(0)=\tilde{a}_{11}^{\mathrm{tr}}, \bar{\partial} \partial \tilde{h}^{-1}(0)=-\tilde{a}_{11}^{\mathrm{tr}}, \bar{\partial}^{2} \partial^{2} \tilde{h}(0)=4 \tilde{a}_{22}^{\mathrm{tr}}$.

Proof. Since $\tilde{K}(z, z)$ is a real analytic function with $\tilde{K}(z, 0)=I$ for $z \in \mathbb{D}$ and $\tilde{h}(z)=$ $\tilde{K}(z, z)^{\operatorname{tr}}$, it follows that $\partial^{m} \tilde{h}(0)=m ! a_{m 0}^{\operatorname{tr}}=0$ and $\bar{\partial}^{n} \tilde{h}(0)=n ! a_{0 n}^{\operatorname{tr}}=0$. By the same token, for $\tilde{h}^{-1}(z)=\tilde{K}^{-1}(z, z)$, we have $\partial^{m} \tilde{h}^{-1}(0)=0$ and $\bar{\partial}^{n} \tilde{h}^{-1}(0)=0$ since $\tilde{K}^{-1}(z, 0)=I$ as well for 
all $z \in \mathbb{D}$. This completes the proof of part (a). To prove part (b), we note that $\tilde{h}(z) \tilde{h}^{-1}(z)=I$ implies $\bar{\partial} \partial \tilde{h}^{-1}(0)=-\tilde{a}_{11}^{\mathrm{tr}}$. Clearly $\bar{\partial} \partial \tilde{h}(0)=\tilde{a}_{11}^{\mathrm{tr}}$ and $\bar{\partial}^{2} \partial^{2} \tilde{h}(0)=4 \tilde{a}_{22}^{\mathrm{tr}}$.

Lemma 2.3. The curvature $\tilde{\mathcal{K}}$ and the covariant derivative of the curvature $\tilde{\mathcal{K}}_{\bar{z}^{n}}$ at 0 are given by the formulae

$$
\tilde{\mathcal{K}}(0)=\tilde{a}_{11}^{\operatorname{tr}} \quad \text { and } \quad \tilde{\mathcal{K}}_{\bar{z}^{n}}(0)=(n+1) ! \mathfrak{a}_{1, n+1}^{\operatorname{tr}} .
$$

Proof. Since $\tilde{\mathcal{K}}(z)=\frac{\partial}{\partial \bar{z}}\left(\tilde{h}^{-1} \frac{\partial}{\partial z} \tilde{h}\right)(z)$, it follows that $\tilde{\mathcal{K}}(0)=\bar{\partial} \tilde{h}^{-1}(0) \partial \tilde{h}(0)+\tilde{h}^{-1}(0) \bar{\partial}$ $\partial \tilde{h}(0)=\tilde{a}_{11}^{\text {tr }}$, by the previous lemma. Also, $\tilde{\mathcal{K}}_{\bar{z}^{n}}(0)=\bar{\partial}^{n} \tilde{\mathcal{K}}(0)=\bar{\partial}^{n+1}\left(\tilde{h}^{-1} \partial \tilde{h}\right)(0)$ (see [7, Proposition 2.17, p. 211]). From Lemma 2.2, we have $\bar{\partial}^{\ell} \tilde{h}^{-1}(0)=0$ for $\ell \geqslant 1$. Therefore, using the Leibnitz rule,

$$
\tilde{\mathcal{K}}_{\bar{z}^{n}}(0)=\sum_{k=0}^{n+1}\left(\begin{array}{c}
n+1 \\
k
\end{array}\right) \bar{\partial}^{n+1-k} \tilde{h}^{-1}(0) \bar{\partial}^{k} \partial \tilde{h}(0)=\bar{\partial}^{n+1} \partial \tilde{h}(0)=(n+1) ! \tilde{a}_{1, n+1}^{\mathrm{tr}} .
$$

This proves the second assertion.

Lemma 2.4. If $\tilde{\mathcal{K}}$ is the curvature of the bundle $(\tilde{E}, \tilde{h})$, then $\tilde{\mathcal{K}}_{z \bar{z}}(0)=2\left(2 \tilde{a}_{22}-\tilde{a}_{11}^{2}\right)^{\operatorname{tr}}$.

Proof. We know from [7, Proposition 2.17, p. 211] that for a bundle map $\Theta$ of a Hermitian holomorphic vector bundle $(\tilde{E}, \tilde{h})$, the covariant derivatives $\Theta_{z}$ and $\Theta_{\bar{z}}$ with respect to a holomorphic frame $f$ are given by $\Theta_{z}(f)=\partial \Theta(f)+\left[\tilde{h}^{-1} \partial \tilde{h}, \Theta(f)\right]$ and $\Theta_{\bar{z}}(f)=\bar{\partial} \Theta(f)$. Since the curvature $\tilde{\mathcal{K}}$ is a bundle map, it follows that

$$
\begin{aligned}
\tilde{\mathcal{K}}_{z \bar{z}}(z) & =\bar{\partial}\left(\partial \tilde{\mathcal{K}}(z)+\left[\tilde{h}^{-1} \partial \tilde{h}, \tilde{\mathcal{K}}\right](z)\right) \\
& =\bar{\partial} \partial \tilde{\mathcal{K}}(z)+\left[\bar{\partial}\left(\tilde{h}^{-1} \partial \tilde{h}\right), \tilde{\mathcal{K}}\right](z)+\left[\tilde{h}^{-1} \partial \tilde{h}, \bar{\partial} \tilde{\mathcal{K}}\right](z) \\
& =\bar{\partial} \partial \tilde{\mathcal{K}}(z)+\left[\tilde{h}^{-1} \partial \tilde{h}, \bar{\partial} \tilde{\mathcal{K}}\right](z) .
\end{aligned}
$$

Since $\partial \tilde{h}(0)=0$ by Lemma 2.2 , we have $\tilde{\mathcal{K}}_{z \bar{z}}(0)=\bar{\partial} \partial \tilde{\mathcal{K}}(0)$. Consequently $\left.\tilde{\mathcal{K}}_{z \bar{z}}(z)\right|_{z=0}=$ $\left.\bar{\partial} \partial\left(\bar{\partial} \tilde{h}^{-1} \partial \tilde{h}\right)(z)\right|_{z=0}$. This simplifies considerably since $\partial \tilde{h}(0)=\partial^{2} \tilde{h}(0)=\partial \tilde{h}^{-1}(0)=0$, again by Lemma 2.2. Thus we obtain

$$
\tilde{\mathcal{K}}_{z \bar{z}}(0)=2 \bar{\partial} \partial \tilde{h}^{-1}(0) \bar{\partial} \partial \tilde{h}(0)+\bar{\partial}^{2} \partial^{2} \tilde{h}(0)=-2 \tilde{a}_{11}^{\operatorname{tr}} \tilde{a}_{11}^{\operatorname{tr}}+4 a_{22}^{\operatorname{tr}}=2\left(2 \tilde{a}_{22}-\tilde{a}_{11}^{2}\right)^{\operatorname{tr}} .
$$

Lemma 2.5. The coefficient of $z^{k+1} \bar{w}^{\ell+1}$ in the power series expansion of $\tilde{K}(z, w)$ is

$$
\begin{aligned}
\tilde{a}_{k+1, \ell+1}= & a_{00}^{1 / 2}\left(\sum_{s=1}^{k} \sum_{t=1}^{\ell} b_{s 0} a_{k+1-s, \ell+1-t} b_{0 t}+\sum_{s=1}^{k} b_{s 0} a_{k+1-s, \ell+1} b_{00}\right. \\
& \left.+\sum_{t=1}^{\ell} b_{00} a_{k+1, \ell+1-t} b_{0 t}+b_{00} a_{k+1, \ell+1} b_{00}-b_{k+1,0} a_{00} b_{0, \ell+1}\right) a_{00}^{1 / 2}
\end{aligned}
$$

for $k, \ell \geqslant 0$. 
Proof. From the definition of $\tilde{K}(z, w)$ we see that for $k, \ell \geqslant 0$

$$
\begin{aligned}
\tilde{a}_{k+1, \ell+1}= & a_{00}^{1 / 2}\left(\sum_{s=0}^{k+1} \sum_{t=0}^{\ell+1} b_{s 0} a_{k+1-s, \ell+1-t} b_{0 t}\right) a_{00}^{1 / 2} \\
= & a_{00}^{1 / 2}\left(\sum_{s=1}^{k+1} \sum_{t=1}^{\ell+1} b_{s 0} a_{k+1-s, \ell+1-t} b_{0 t}+\sum_{s=1}^{k+1} b_{s 0} a_{k+1-s, \ell} b_{00}\right. \\
& \left.+\sum_{t=1}^{\ell+1} b_{00} a_{k+1, \ell+1-t} b_{0 t}+b_{00} a_{k+1, \ell+1} b_{00}\right) a_{00}^{1 / 2} \\
= & a_{00}^{1 / 2}\left(\sum_{s=1}^{k} \sum_{t=1}^{\ell} b_{s 0} a_{k+1-s, \ell+1-t} b_{0 t}+\sum_{s=1}^{k+1} b_{s 0} a_{k+1-s, 0} b_{0, \ell+1}+\sum_{t=1}^{\ell} b_{k+1,0} a_{0, \ell+1-t} b_{0 t}\right. \\
& \left.+\sum_{s=1}^{k+1} b_{s 0} a_{k+1-s, \ell} b_{00}+\sum_{t=1}^{\ell+1} b_{00} a_{k+1, \ell+1-t} b_{0 t}+b_{00} a_{k+1, \ell+1} b_{00}\right) a_{00}^{1 / 2} \\
= & a_{00}^{1 / 2}\left(\sum_{s=1}^{k} \sum_{t=1}^{\ell} b_{s 0} a_{k+1-s, \ell+1-t} b_{0 t}+\left(\sum_{s=0}^{k+1} b_{s 0} a_{k+1-s, 0}\right) b_{0, \ell+1}\right. \\
& +b_{k+1,0}\left(\sum_{t=0}^{\ell+1} a_{0, \ell+1-t} b_{0 t}\right)+\sum_{s=1}^{k} b_{s 0} a_{k+1-s, \ell} b_{00}+\sum_{t=1}^{\ell} b_{00} a_{k+1, \ell+1-t} b_{0 t} \\
& \left.+b_{00} a_{k+1, \ell+1} b_{00}-b_{k+1,0} a_{00} b_{0, \ell+1}\right) a_{00}^{1 / 2} \\
= & a_{00}^{1 / 2}\left(\sum_{s=1}^{k} \sum_{t=1}^{\ell} b_{s 0} a_{k+1-s, \ell+1-t} b_{0 t}+\sum_{s=1}^{k} b_{s 0} a_{k+1-s, \ell} b_{00}+\sum_{t=1}^{\ell} b_{00} a_{k+1, \ell+1-t} b_{0 t}\right. \\
& \left.+b_{00} a_{k+1, \ell+1} b_{00}-b_{k+1,0} a_{00} b_{0, \ell+1}\right) a_{00}^{1 / 2}
\end{aligned}
$$

as the coefficient of $z^{k+1}$ in $K(z, w)^{-1} K(z, w)=\sum_{s=0}^{k+1} b_{s 0} a_{k+1-s, 0}=0$ and the coefficient of $\bar{w}^{\ell+1}$ in $K(z, w) K(z, w)^{-1}=\sum_{t=0}^{\ell+1} a_{0, \ell+1-t} b_{0 t}=0$ for $k, \ell \geqslant 0$.

The following theorem will be useful in the sequel. For $T$ in $\mathrm{B}_{n}(\Omega)$, recall that $\mathcal{K}_{T}$ denotes the curvature of the bundle $E_{T}$ corresponding to $T$.

Theorem 2.6. Suppose that $T_{1}$ and $T_{2}$ are homogeneous operators in $\mathrm{B}_{n}(\mathbb{D})$. Then $\mathcal{K}_{T_{1}}(0)$ and $\left(\mathcal{K}_{T_{1}}\right)_{\bar{z}}(0)$ are simultaneously unitarily equivalent to $\mathcal{K}_{T_{2}}(0)$ and $\left(\mathcal{K}_{T_{2}}\right)_{\bar{z}}(0)$, respectively, if and only if $\mathcal{K}_{T_{1}}(z)$ and $\left(\mathcal{K}_{T_{1}}\right)_{\bar{z}}(z)$ are simultaneously unitarily equivalent to $\mathcal{K}_{T_{2}}(z)$ and $\left(\mathcal{K}_{T_{2}}\right)_{\bar{z}}(z)$, respectively, for all $z$ in $\mathbb{D}$.

Notation 2.7. Before going into the proof of Theorem 2.6, let us fix some notation. Let

$$
\text { Möb }=\left\{\varphi_{t, a}: t \in \mathbb{T}, a \in \mathbb{D}\right\}, \quad \text { where } \varphi_{t, a}(z)=t \frac{z-a}{1-\bar{a} z} .
$$

Möb is the group of biholomorphic automorphisms of the unit disc $\mathbb{D}$. Let $c:$ Möb $\times \mathbb{D} \rightarrow \mathbb{C}$ be the function which is given by the formula

$$
c\left(\varphi^{-1}, z\right):=\left(\varphi^{-1}\right)^{\prime}(z),
$$


where the prime stands for differentiation with respect to $z$. The function $c$ satisfies the following cocycle property:

$$
c\left(\varphi^{-1} \psi^{-1}, z\right)=c\left(\varphi^{-1}, \psi^{-1}(z)\right) c\left(\psi^{-1}, z\right), \quad \text { for } \varphi \in \text { Möband } z \in \mathbb{D} .
$$

The cocycle property can be easily verified by the chain rule.

Lemma 2.8. Suppose that $T$ in $\mathrm{B}_{n}(\mathbb{D})$ is homogeneous. Then we have:

(a) $\mathcal{K}_{T}\left(\varphi^{-1}(0)\right)=\left|c\left(\varphi^{-1}, 0\right)\right|^{-2} U_{\varphi}^{-1} \mathcal{K}_{T}(0) U_{\varphi}$;

(b) $\left(\mathcal{K}_{T}\right)_{\bar{z}}\left(\varphi^{-1}(0)\right)=\left|c\left(\varphi^{-1}, 0\right)\right|^{-2} \overline{c\left(\varphi^{-1}, 0\right)^{-1}} U_{\varphi}^{-1}\left(\left(\mathcal{K}_{T}\right)_{\bar{z}}(0)-\overline{c\left(\varphi^{-1}, 0\right)^{-1}\left(\varphi^{-1}\right)^{(2)}(0)} \mathcal{K}_{T}(0)\right)$ $U_{\varphi}$

for some unitary operator $U_{\varphi}, \varphi \in$ Möb.

Proof. Following [7], using the homogeneity of the operator $T$, we find that there is a unitary operator $U_{\varphi, z}$ such that

$$
\mathcal{K}_{\varphi(T)}(z)=U_{\varphi, z}^{-1} \mathcal{K}_{T}(z) U_{\varphi, z}, \quad \varphi \in \text { Möb and } z \in \mathbb{D} .
$$

On the other hand, an application of the chain rule gives the formula

$$
\mathcal{K}_{\varphi(T)}(z)=\left|\left(\varphi^{-1}\right)^{\prime}(z)\right|^{2} \mathcal{K}_{T}\left(\left(\varphi^{-1}\right)(z)\right) \quad \text { for } \varphi \in \text { Möb and } z \in \mathbb{D} \text {. }
$$

Putting both of these together, we clearly have

$$
U_{\varphi, z}^{-1} \mathcal{K}_{T}(z) U_{\varphi, z}=\left|c\left(\varphi^{-1}, z\right)\right|^{2} \mathcal{K}_{T}\left(\left(\varphi^{-1}\right)(z)\right) .
$$

In particular, if $z=0$, then

$$
U_{\varphi, 0}^{-1} \mathcal{K}_{T}\left(\varphi^{-1}(0)\right) U_{\varphi, 0}=\left|c\left(\varphi^{-1}, 0\right)\right|^{2} \mathcal{K}_{T}\left(\left(\varphi^{-1}\right)(0)\right) .
$$

Set $U_{\varphi, 0}:=U_{\varphi}$. Then

$$
\mathcal{K}_{T}\left(\varphi^{-1}(0)\right)=\left|c\left(\varphi^{-1}, 0\right)\right|^{-2} U_{\varphi}^{-1} \mathcal{K}_{T}(0) U_{\varphi}
$$

for $\varphi \in$ Möb, $z \in \mathbb{D}$. This proves part (a).

To prove part (b), we differentiate $\mathcal{K}_{\varphi(T)}$ with respect to $\bar{z}$ using (2.3) to see that

$$
\begin{aligned}
\bar{\partial} \mathcal{K}_{\varphi(T)}(z) & =\left(\varphi^{-1}\right)^{\prime}(z) \overline{\left(\varphi^{-1}\right)^{(2)}(z)} \mathcal{K}_{T}\left(\varphi^{-1}(z)\right)+\left|\left(\varphi^{-1}\right)^{\prime}(z)\right|^{2} \overline{\left(\varphi^{-1}\right)^{\prime}(z)} \bar{\partial} \mathcal{K}_{T}\left(\varphi^{-1}(z)\right) \\
& =c\left(\varphi^{-1}, z\right) \overline{\left(\varphi^{-1}\right)^{(2)}(z)} \mathcal{K}_{T}\left(\varphi^{-1}(z)\right)+\left|c\left(\varphi^{-1}, z\right)\right|^{2} \overline{c\left(\varphi^{-1}, z\right)} \bar{\partial} \mathcal{K}_{T}\left(\varphi^{-1}(z)\right) .
\end{aligned}
$$

Using (2.4) and (a), putting $z=0$ and $U_{\varphi, 0}=U_{\varphi}$, we see that

$$
\begin{aligned}
U_{\varphi}^{-1} \bar{\partial} \mathcal{K}_{T}(0) U_{\varphi}= & c\left(\varphi^{-1}, 0\right) \overline{\left(\varphi^{-1}\right)^{(2)}(0)} \mathcal{K}_{T}\left(\varphi^{-1}(0)\right)+\left|c\left(\varphi^{-1}, 0\right)\right|^{2} \overline{c\left(\varphi^{-1}, 0\right)} \bar{\partial} \mathcal{K}_{T}\left(\varphi^{-1}(0)\right) \\
= & c\left(\varphi^{-1}, 0\right) \overline{\left(\varphi^{-1}\right)^{(2)}(0)}\left|c\left(\varphi^{-1}, 0\right)\right|^{-2} U_{\varphi}^{-1} \mathcal{K}_{T}(0) U_{\varphi} \\
& +\left|c\left(\varphi^{-1}, 0\right)\right|^{2} \overline{c\left(\varphi^{-1}, 0\right)} \bar{\partial} \mathcal{K}_{T}\left(\varphi^{-1}(0)\right) \\
= & \overline{c\left(\varphi^{-1}, 0\right)^{-1}\left(\varphi^{-1}\right)^{(2)}(0)} U_{\varphi}^{-1} \mathcal{K}_{T}(0) U_{\varphi}+\left|c\left(\varphi^{-1}, 0\right)\right|^{2} \overline{c\left(\varphi^{-1}, 0\right)} \bar{\partial} \mathcal{K}_{T}\left(\varphi^{-1}(0)\right) .
\end{aligned}
$$

Therefore

$$
\bar{\partial} \mathcal{K}_{T}\left(\varphi^{-1}(0)\right)=\left|c\left(\varphi^{-1}, 0\right)\right|^{-2} \overline{c\left(\varphi^{-1}, 0\right)^{-1}} U_{\varphi}^{-1}\left(\bar{\partial} \mathcal{K}_{T}(0)-\overline{c\left(\varphi^{-1}, 0\right)^{-1}\left(\varphi^{-1}\right)^{(2)}(0)} \mathcal{K}_{T}(0)\right) U_{\varphi} .
$$

The proof of part (b) is complete since $\left(\mathcal{K}_{T}\right)_{\bar{z}}=\bar{\partial} \mathcal{K}_{T}$ (cf. [7]).

Corollary 2.9. Suppose that $T_{1}, T_{2}$ are homogeneous operators in $\mathrm{B}_{n}(\mathbb{D})$. Then

$$
\text { (1) } U^{-1} \mathcal{K}_{T_{2}}(0) U=\mathcal{K}_{T_{1}}(0), \quad(2) U^{-1}\left(\mathcal{K}_{T_{2}}\right)_{\bar{z}}(0) U=\left(\mathcal{K}_{T_{1}}\right)_{\bar{z}}(0)
$$


for some unitary operator $U$ if and only if

$$
\text { (i) } V_{\varphi}^{-1} \mathcal{K}_{T_{2}}(z) V_{\varphi}=\mathcal{K}_{T_{1}}(z), \quad \text { (ii) } V_{\varphi}^{-1}\left(\mathcal{K}_{T_{2}}\right)_{\bar{z}}(z) V_{\varphi}=\left(\mathcal{K}_{T_{1}}\right)_{\bar{z}}(z)
$$

for some unitary operator $V_{\varphi}, \varphi$ in Möb and $z \in \mathbb{D}$.

Proof. The 'if' part is obvious. To prove the 'only if' part, take $\varphi=\varphi_{t, z}$, where $\varphi_{t, a}(w)=$ $t(w-a) /(1-\bar{a} w)$, for $a, w \in \mathbb{D}$ and $t \in \mathbb{T}$. Choose a unitary operator such that (a) and (b) of Lemma 2.8 are satisfied. We get from (1) and Lemma 2.8(a) that

$$
\begin{aligned}
\mathcal{K}_{T_{1}}(z) & =\left|c\left(\varphi^{-1}, 0\right)\right|^{-2} U_{\varphi}^{-1} \mathcal{K}_{T_{1}}(0) U_{\varphi} \\
& =\left|c\left(\varphi^{-1}, 0\right)\right|^{-2} U_{\varphi}^{-1} U^{-1} \mathcal{K}_{T_{2}}(0) U U_{\varphi} \\
& =\left|c\left(\varphi^{-1}, 0\right)\right|^{-2} U_{\varphi}^{-1} U^{-1}\left|c\left(\varphi^{-1}, 0\right)\right|^{2} U_{\varphi} \mathcal{K}_{T_{2}}(z) U_{\varphi}^{-1} U U_{\varphi} \\
& =U_{\varphi}^{-1} U^{-1} U_{\varphi} \mathcal{K}_{T_{2}}(z) U_{\varphi}^{-1} U U_{\varphi} .
\end{aligned}
$$

Since $V_{\varphi}:=U_{\varphi}^{-1} U U_{\varphi}$ is unitary, the proof of (i) is complete.

From (1), (2) and Lemma 2.8(b)

$$
\begin{aligned}
\left(\mathcal{K}_{T_{1}}\right)_{\bar{z}}(z)= & \left|c\left(\varphi^{-1}, 0\right)\right|^{-2} \overline{c\left(\varphi^{-1}, 0\right)^{-1}} U_{\varphi}^{-1}\left(\left(\mathcal{K}_{T_{1}}\right)_{\bar{z}}(0)-\overline{c\left(\varphi^{-1}, 0\right)^{-1}\left(\varphi^{-1}\right)^{(2)}(0)} \mathcal{K}_{T_{1}}(0)\right) U_{\varphi} \\
= & \left|c\left(\varphi^{-1}, 0\right)\right|^{-2} \overline{c\left(\varphi^{-1}, 0\right)^{-1}} U_{\varphi}^{-1} U^{-1}\left(\left(\mathcal{K}_{T_{2}}\right)_{\bar{z}}(0)-\overline{c\left(\varphi^{-1}, 0\right)^{-1}\left(\varphi^{-1}\right)^{(2)}(0)} \mathcal{K}_{T_{2}}(0)\right) U U_{\varphi} \\
= & \left|c\left(\varphi^{-1}, 0\right)\right|^{-2} \overline{c\left(\varphi^{-1}, 0\right)^{-1}} U_{\varphi}^{-1} U^{-1}\left(\overline{\left(\varphi^{-1}\right)^{(2)}(0) c\left(\varphi^{-1}, 0\right)^{-1}} \mathcal{K}_{T_{2}}(0)\right. \\
& \left.+\left|c\left(\varphi^{-1}, 0\right)\right|^{2} \overline{c\left(\varphi^{-1}, 0\right)} U_{\varphi}\left(\mathcal{K}_{T_{2}}\right)_{\bar{z}}(z) U_{\varphi}^{-1}-\overline{\left(\varphi^{-1}\right)^{(2)}(0) c\left(\varphi^{-1}, 0\right)^{-1}} \mathcal{K}_{T_{2}}(0)\right) U U_{\varphi} \\
= & U_{\varphi}^{-1} U^{-1} U_{\varphi}\left(\mathcal{K}_{T_{2}}\right)_{\bar{z}}(z) U_{\varphi}^{-1} U U_{\varphi} .
\end{aligned}
$$

Taking $V_{\varphi}=U_{\varphi}^{-1} U U_{\varphi}$ as before, we have (ii).

Proof of Theorem 2.6. Combining Lemma 2.8 and Corollary 2.9, we get the proof of Theorem 2.6.

Notation 2.10. For a positive integer $m$, let $S\left(c_{1}, \ldots, c_{m}\right)$ denote the forward shift on $\mathbb{C}^{m+1}$ with weight sequence $\left(c_{1}, \ldots, c_{m}\right), c_{i} \in \mathbb{C}$, that is,

$$
S\left(c_{1}, \ldots, c_{m}\right)(\ell, p)=c_{\ell} \delta_{p+1, \ell} \quad \text { for } 0 \leqslant p, \ell \leqslant m .
$$

We set $\mathbb{S}_{m}:=S(1, \ldots, m)$. For $A$ in $\mathcal{M}_{p, q}$, we let $A(i, j)$ denote the $(i, j)$ th entry of the matrix $A$ for $1 \leqslant i \leqslant p, 1 \leqslant j \leqslant q$. For a vector $\boldsymbol{v}$ in $\mathbb{C}^{k}$, let $\boldsymbol{v}(i)$ denote the $i$ th component of the vector $\boldsymbol{v}, 1 \leqslant i \leqslant k$.

EXAmple 2.11. From (2.1), we get

$$
B_{1}^{\left(\alpha, \beta^{\prime}\right)}(z, w)=\left(\begin{array}{cc}
(1-z \bar{w})^{2} & \beta^{\prime} z(1-z \bar{w}) \\
\beta^{\prime} \bar{w}(1-z \bar{w}) & \beta^{\prime}\left(1+\beta^{\prime} z \bar{w}\right)
\end{array}\right)(1-z \bar{w})^{-\alpha-\beta^{\prime}-2}
$$

and

$$
B_{2}^{(\alpha, \beta)}(z, w)=\left(\begin{array}{ccc}
(1-z \bar{w})^{4} & \beta(1-z \bar{w})^{3} z & \beta(\beta+1)(1-z \bar{w})^{2} z^{2} \\
\beta(1-z \bar{w})^{3} \bar{w} & \beta(1+\beta z \bar{w})(1-z \bar{w})^{2} & \beta(\beta+1)(2+\beta z \bar{w}) \\
\beta(\beta+1) & \beta(\beta+1)(2+\beta z \bar{w}) & \beta(\beta+1)(2+(\beta+1) \\
(1-z \bar{w})^{2} \bar{w}^{2} & (1-z \bar{w}) \bar{w} & (4+\beta z \bar{w}) z \bar{w})
\end{array}\right)(1-z \bar{w})^{-\alpha-\beta-4}
$$


for $\quad \alpha, \beta, \beta^{\prime}>0 \quad$ and $\quad(z, w) \in \mathbb{D} \times \mathbb{D}$. Let $\quad K_{1}(z, w):=(1-z \bar{w})^{-\alpha} \oplus B_{1}^{\left(\alpha, \beta^{\prime}\right)}(z, w)$ and $K_{2}(z, w):=B_{2}^{\left(\alpha, \beta^{\prime}\right)}(z, w)$ for $(z, w) \in \mathbb{D} \times \mathbb{D}$. Let $M_{1}$ and $M_{2}$ be the multiplication operators on the Hilbert spaces $\mathcal{H}_{1}$ and $\mathcal{H}_{2}$ with reproducing kernels $K_{1}$ and $K_{2}$, respectively. Clearly, $M_{1}$ is the direct sum $M^{(\alpha)} \oplus M_{1}^{\left(\alpha, \beta^{\prime}\right)}$ acting on the Hilbert space $\mathbb{A}^{(\alpha)} \oplus A_{1}^{\left(\alpha, \beta^{\prime}\right)}$ and $M_{2}$ is the multiplication operator on the Hilbert space $A_{2}^{(\alpha, \beta)}$. Wilkins [19] has shown that the adjoint of the operator $M_{1}^{\left(\alpha, \beta^{\prime}\right)}$ on $A_{1}^{\left(\alpha, \beta^{\prime}\right)}$ is in $\mathrm{B}_{2}(\mathbb{D})$. This operator is also homogeneous. It is easy to see that the operator $M^{(\alpha)}$ is homogeneous and its adjoint is in the class $\mathrm{B}_{1}(\mathbb{D})$. Consequently, the direct sum, namely, $M_{1}^{*}$ is homogeneous and lies in the class $\mathrm{B}_{3}(\mathbb{D})$. The operator $M_{2}^{*}$ is in $\mathrm{B}_{3}(\mathbb{D})$ by $[\mathbf{1 2}$, Proposition 3.6] and is homogeneous by [4, p. 428] and [16, Theorem 5.1]. Let

$$
h_{1}(z)=K_{1}(z, z)^{\operatorname{tr}} \quad \text { and } \quad h_{2}(z)=K_{2}(z, z)^{\operatorname{tr}} .
$$

Thus $h_{1}$ and $h_{2}$ are the metrics for the bundles $E_{1}$ and $E_{2}$ corresponding to the operators $M_{1}^{*}$ and $M_{2}^{*}$, respectively.

Lemma 2.12. The curvature at zero and the covariant derivatives of curvature at zero of order $(0,1)$ and $(1,1)$ for the bundles $E_{1}$ and $E_{2}$ are

(a) $\tilde{\mathcal{K}}_{1}(0)=\operatorname{diag}\left(\alpha, \alpha, \alpha+2 \beta^{\prime}+2\right), \quad\left(\tilde{\mathcal{K}}_{1}\right)_{\bar{z}}(0)=S\left(0,-2 \sqrt{\beta^{\prime}}\left(\beta^{\prime}+1\right)\right)^{\text {tr }}$ and $\left(\tilde{\mathcal{K}}_{1}\right)_{z \bar{z}}(0)=$ $2 \operatorname{diag}\left(\alpha, \alpha+\beta^{\prime}\left(\beta^{\prime}+1\right), \alpha+\beta^{\prime}\left(-\beta^{\prime}+1\right)+2\right) ;$

(b) $\tilde{\mathcal{K}}_{2}(0)=\operatorname{diag}(\alpha, \alpha, \alpha+3 \beta+6),\left(\tilde{\mathcal{K}}_{2}\right)_{\bar{z}}(0)=S(0,-3 \sqrt{2(\beta+1)}(\beta+2))^{\operatorname{tr}}$ and $\left(\tilde{\mathcal{K}}_{2}\right)_{z \bar{z}}(0)=$ $\operatorname{diag}(\alpha, \alpha+3(\beta+1)(\beta+2), \alpha-3 \beta(\beta+2))$,

respectively. Here $\tilde{\mathcal{K}}_{i},\left(\tilde{\mathcal{K}}_{i}\right)_{\bar{z}}$ and $\left(\tilde{\mathcal{K}}_{i}\right)_{z \bar{z}}$ are computed with respect to the metrics $\tilde{h}_{i}$ for $i=1,2$ obtained from the corresponding reproducing kernels normalized at 0.

(If $\tilde{h}$ is a metric corresponding to a normalized reproducing kernel at 0 , then $\tilde{h}(0)=I$, that is, the basis for the fibre at 0 with respect to which $\tilde{h}(0)$ is computed is orthonormal.)

Proof. For any reproducing kernel $K$ with

$$
K(z, w)=\sum_{m, n \geqslant 0} a_{m n} z^{m} \bar{w}^{n} \text { and } \quad K(z, w)^{-1}=\sum_{m, n \geqslant 0} b_{m n} z^{m} \bar{w}^{n}
$$

the identity $K(z, w)^{-1} K(z, w)=I$ implies that

$$
b_{00}=a_{00}^{-1} \text { and } \sum_{\ell=0}^{k} b_{0, k-\ell} a_{0 \ell}=0, k \geqslant 1 .
$$

For $k=1$, we have $b_{10}=-a_{00}^{-1} a_{10} a_{00}^{-1}, b_{01}=\left(b_{10}\right)^{*}$. Also, by Lemma 2.5 , we have

$$
a_{11}=a_{00}^{1 / 2}\left(b_{00} a_{11} b_{00}-b_{10} a_{00} b_{01}\right) a_{00}^{1 / 2}=a_{00}^{-1 / 2}\left(a_{11}-a_{10} a_{00}^{-1} a_{01}\right) a_{00}^{-1 / 2} .
$$

For $k=2$, we have $b_{02}=-\left(b_{01} a_{01}+b_{00} a_{02}\right) a_{00}^{-1}=a_{00}^{-1}\left(a_{01} a_{00}^{-1} a_{01}-a_{02}\right) a_{00}^{-1}$. Now, Lemma 2.5 gives

$$
\begin{aligned}
\tilde{a}_{12} & =a_{00}^{1 / 2}\left(b_{00} a_{11} b_{01}+b_{00} a_{12} b_{00}-b_{10} a_{00} b_{02}\right) a_{00}^{1 / 2} \\
& =a_{00}^{-1 / 2}\left(a_{12}-\left(a_{11}-a_{10} a_{00}^{-1} a_{01}\right) a_{00}^{-1} a_{01}-a_{10} a_{00}^{-1} a_{02}\right) a_{00}^{-1 / 2} .
\end{aligned}
$$


Observing that $b_{20}=b_{02}{ }^{*}=a_{00}^{-1}\left(a_{10} a_{00}^{-1} a_{10}-a_{20}\right) a_{00}^{-1}$, from Lemma 2.5, we have

$$
\begin{aligned}
\tilde{a}_{22}= & a_{00}^{1 / 2}\left(b_{10} a_{11} b_{01}+b_{10} a_{12} b_{00}+b_{00} a_{21} b_{01}+b_{00} a_{22} b_{00}-b_{20} a_{00} b_{02}\right) a_{00}^{1 / 2} \\
= & a_{00}^{-1 / 2}\left(a_{10} a_{00}^{-1} a_{11} a_{00}^{-1} a_{01}-a_{10} a_{00}^{-1} a_{12}-a_{21} a_{00}^{-1} a_{01}+a_{22}\right. \\
& \left.-\left(a_{10} a_{00}^{-1} a_{10}-a_{20}\right) a_{00}^{-1}\left(a_{01} a_{00}^{-1} a_{01}-a_{02}\right)\right) a_{00}^{-1 / 2} \\
= & a_{00}^{-1 / 2}\left(a_{22}+\left(a_{20} a_{00}^{-1} a_{01}-a_{21}\right) a_{00}^{-1} a_{01}-a_{20} a_{00}^{-1} a_{02}\right. \\
& \left.-a_{10} a_{00}^{-1}\left(a_{12}-\left(a_{11}-a_{10} a_{00}^{-1} a_{01}\right) a_{00}^{-1} a_{01}-a_{10} a_{00}^{-1} a_{02}\right)\right) a_{00}^{-1 / 2} .
\end{aligned}
$$

In particular, choosing $K=K_{1}$, we have

$$
\begin{gathered}
a_{00}=\operatorname{diag}\left(1,1, \beta^{\prime}\right), \quad a_{01}=S\left(0, \beta^{\prime}\right) \\
a_{11}=\operatorname{diag}\left(\alpha, \alpha+\beta^{\prime}, \beta^{\prime}\left(\alpha+2 \beta^{\prime}+2\right)\right), \quad a_{12}=S\left(0, \beta^{\prime}\left(\alpha+\beta^{\prime}+1\right)\right) ; \\
a_{22}=\operatorname{diag}\left(\frac{\alpha(\alpha+1)}{2}, \frac{\left(\alpha+\beta^{\prime}\right)\left(\alpha+\beta^{\prime}+1\right)}{2}, \frac{\beta^{\prime}\left(\alpha+\beta^{\prime}+2\right)\left(\alpha+3 \beta^{\prime}+3\right)}{2}\right)
\end{gathered}
$$

and

$$
a_{20}=0 .
$$

Thus $a_{11}-a_{10} a_{00}^{-1} a_{01}=\operatorname{diag}\left(\alpha, \alpha, \beta^{\prime}\left(\alpha+2 \beta^{\prime}+2\right)\right)$. Hence from Lemma 2.3 and equation (2.8), we have $\tilde{\mathcal{K}}_{1}(0)=\tilde{a}_{11}^{\text {tr }}=\operatorname{diag}\left(\alpha, \alpha, \alpha+2 \beta^{\prime}+2\right)$.

From equation (2.9), we get $\tilde{a}_{12}=S\left(0,-\sqrt{\beta^{\prime}}\left(\beta^{\prime}+1\right)\right)$. Therefore, from Lemma 2.3 , we have $\left(\tilde{\mathcal{K}}_{1}\right)_{\bar{z}}(0)=2 \tilde{a}_{12}^{\operatorname{tr}}=S\left(0,-2 \sqrt{\beta^{\prime}}\left(\beta^{\prime}+1\right)\right)^{\operatorname{tr}}$.

Similarly, from equation (2.10), $\quad \tilde{a}_{22}=\operatorname{diag}\left(\alpha(\alpha+1) / 2,\left(\alpha(\alpha+1)+\beta^{\prime}\left(\beta^{\prime}+1\right)\right) / 2\right.$, $\left.\left(\alpha+\beta^{\prime}+2\right)\left(\alpha+3 \beta^{\prime}+3\right) / 2\right)$. Hence

$$
\left(\tilde{\mathcal{K}}_{1}\right)_{z \bar{z}}(0)=2\left(2 \tilde{a}_{22}-\tilde{a}_{11}^{2}\right)^{\operatorname{tr}}=2 \operatorname{diag}\left(\alpha, \alpha+\beta^{\prime}\left(\beta^{\prime}+1\right), \alpha+\beta^{\prime}\left(-\beta^{\prime}+1\right)+2\right)
$$

from Lemma 2.4. This completes the proof of (a).

To prove (b), choose $K=K_{2}$ and observe that

$$
\begin{gathered}
a_{00}=\operatorname{diag}(1, \beta, 2 \beta(\beta+1)), \quad a_{10}=S(\beta, 2 \beta(\beta+1))^{\operatorname{tr}}, \\
a_{12}=S(\beta(\alpha+\beta+1), \beta(\beta+1)(2 \alpha+3 \beta+6)), \quad\left(a_{02}\right)(i, j)= \begin{cases}\beta(\beta+1) & \text { for } i=3, j=1, \\
0 & \text { otherwise, }\end{cases} \\
a_{11}=\operatorname{diag}(\alpha+\beta, \beta(\alpha+2 \beta+2), 2 \beta(\beta+1)(\alpha+3 \beta+6))
\end{gathered}
$$

and

$$
\begin{aligned}
a_{22}= & \operatorname{diag}\left(\frac{(\alpha+\beta)(\alpha+\beta+1)}{2}, \frac{\beta(\alpha+\beta+2)(\alpha+3 \beta+3)}{2}, \beta(\beta+1)((\alpha+\beta+4)(\alpha+\beta+5)\right. \\
& +4(\beta+1)(\alpha+\beta+4)+\beta(\beta+1))) .
\end{aligned}
$$

Therefore $a_{11}-a_{10} a_{00}^{-1} a_{01}=\operatorname{diag}(\alpha, \alpha \beta, 2 \beta(\beta+1)(\alpha+3 \beta+6))$. Hence from Lemma 2.3 and equation (2.8), we have

$$
\tilde{\mathcal{K}}_{2}(0)=\tilde{a}_{11}^{\operatorname{tr}}=\operatorname{diag}(\alpha, \alpha, \alpha+3 \beta+6) .
$$

Also, from equation (2.9), we have

$$
\tilde{a}_{12}=S\left(0,-\frac{3}{\sqrt{2}} \sqrt{\beta+1}(\beta+2)\right)
$$

and from Lemma 2.3, we have

$$
\left(\tilde{\mathcal{K}}_{2}\right)_{\bar{z}}(0)=2 \tilde{a}_{12}^{\mathrm{tr}}=S(0,-3 \sqrt{2(\beta+1)}(\beta+2))^{\operatorname{tr}} .
$$


Since

$$
\tilde{a}_{22}=\operatorname{diag}\left(\frac{\alpha(\alpha+1)}{2}, \frac{\alpha(\alpha+1)+3(\beta+1)(\beta+2)}{2}, \frac{\alpha(\alpha+1)}{2}+3(\beta+2)(\alpha+\beta+3)\right),
$$

from equation (2.10), using Lemma 2.4, we get

$$
\left(\tilde{\mathcal{K}}_{2}\right)_{z \bar{z}}(0)=2\left(2 \tilde{a}_{22}-\tilde{a}_{11}^{2}\right)^{\operatorname{tr}}=2 \operatorname{diag}(\alpha, \alpha+3(\beta+1)(\beta+2), \alpha-3 \beta(\beta+2)) .
$$

By means of a sequence of lemmas proved below, we construct a unitary operator between the vector spaces $\left(\left(E_{1}\right)_{0}, h_{1}(0)\right)$ and $\left(\left(E_{2}\right)_{0}, h_{2}(0)\right)$ which intertwines $\tilde{\mathcal{K}}_{1}(0), \tilde{\mathcal{K}}_{2}(0)$ and $\left(\tilde{\mathcal{K}}_{1}\right)_{\bar{z}}(0),\left(\tilde{\mathcal{K}}_{2}\right)_{\bar{z}}(0)$. Here $\left(E_{1}\right)_{0}$ and $\left(E_{2}\right)_{0}$ are the fibres over 0 of the corresponding bundles $E_{1}$ and $E_{2}$, respectively.

Lemma 2.13. A linear transformation $U_{0}:\left(\mathbb{C}^{3}, h_{2}(0)\right) \rightarrow\left(\mathbb{C}^{3}, h_{1}(0)\right)$ is diagonal and unitary with $U_{0}=\operatorname{diag}\left(u_{1}, u_{2}, u_{3}\right), u_{i} \in \mathbb{C}$ for $i=1,2,3$, if and only if $\left|u_{1}\right|^{2}=1,\left|u_{2}\right|^{2}=\beta,\left|u_{3}\right|^{2}=$ $2 \beta(\beta+1) / \beta^{\prime}$.

Proof. The 'only if' part: Since $U_{0}$ is a unitary operator we have $U_{0}^{*}=U_{0}^{-1}$, where $*$ denotes the adjoint of $U_{0}$. Now, from $[\mathbf{1 2}$, p. 395]

$$
\begin{aligned}
U_{0}^{*} & =h_{2}(0)^{-1} \bar{U}_{0}^{\operatorname{tr}} h_{1}(0) \\
& =\operatorname{diag}\left(1, \beta^{-1},(2 \beta(\beta+1))^{-1}\right) \operatorname{diag}\left(\bar{u}_{1}, \bar{u}_{2}, \bar{u}_{3}\right) \operatorname{diag}\left(1,1, \beta^{\prime}\right) \\
& =\operatorname{diag}\left(\bar{u}_{1}, \frac{\bar{u}_{2}}{\beta}, \frac{\bar{u}_{3} \beta^{\prime}}{2 \beta(\beta+1)}\right) \\
& =\operatorname{diag}\left(u_{1}^{-1}, u_{2}^{-1}, u_{3}^{-1}\right)
\end{aligned}
$$

This implies the desired equalities.

The 'if' part: Taking $u_{1}=1, u_{2}=\sqrt{\beta}, u_{3}=\sqrt{\frac{2 \beta(\beta+1)}{\beta^{\prime}}}$, we see that $U_{0}=\operatorname{diag}\left(u_{1}, u_{2}, u_{3}\right)$ is a unitary operator between the two given vector spaces.

The proof of the next lemma is just a routine verification.

Lemma 2.14. Suppose that $T$ and $\tilde{T}$ are in $\mathcal{M}_{3}$ such that

$$
T(i, j)=\left\{\begin{array}{ll}
\eta & \text { for } i=2, j=3, \\
0 & \text { otherwise. }
\end{array} \quad \text { and } \quad \tilde{T}(i, j)= \begin{cases}\tilde{\eta} & \text { for } i=2, j=3 \\
0 & \text { otherwise }\end{cases}\right.
$$

Then $A T=\tilde{T} A$ for some invertible diagonal matrix $A=\operatorname{diag}\left(a_{1}, a_{2}, a_{3}\right)$ if and only if $\tilde{\eta} / \eta=a_{2} / a_{3}$.

Lemma 2.15. If $\beta^{\prime}=\frac{3}{2} \beta+2$, then $U_{0}^{-1} \tilde{\mathcal{K}}_{1}(0) U_{0}=\tilde{\mathcal{K}}_{2}(0)$ and $U_{0}^{-1}\left(\tilde{\mathcal{K}}_{1}\right)_{\bar{z}}(0) U_{0}=\left(\tilde{\mathcal{K}}_{2}\right)_{\bar{z}}(0)$, where $U_{0}:\left(\mathbb{C}^{3}, h_{2}(0)\right) \rightarrow\left(\mathbb{C}^{3}, h_{1}(0)\right)$, is a diagonal unitary with $U_{0}=\operatorname{diag}\left(u_{1}, u_{2}, u_{3}\right), u_{i} \in \mathbb{C}$ for $i=1,2,3$.

Proof. Our the choice of $\beta^{\prime}$ together with Lemma 2.12 ensures that $\tilde{\mathcal{K}}_{1}(0)=\tilde{\mathcal{K}}_{2}(0)$. The first equality is therefore evident.

Clearly, $\left(\tilde{\mathcal{K}}_{2}\right)_{\bar{z}}(0)$ and $\left(\tilde{\mathcal{K}}_{1}\right)_{\bar{z}}(0)$ are of the form $T$ and $\tilde{T}$ of the previous lemma. Choose

$$
u_{1}=1, \quad u_{2}=\sqrt{\beta}, \quad u_{3}=\sqrt{\frac{2 \beta(\beta+1)}{\beta^{\prime}}} \text { with } \beta^{\prime}=\frac{3}{2} \beta+2 .
$$


To complete the proof of the second equality, by Lemma 2.14, we only have to verify $\tilde{\eta} / \eta=u_{2} / u_{3}$, where $\eta=-3 \sqrt{2(\beta+1)}(\beta+2), \tilde{\eta}=-2 \sqrt{\beta^{\prime}}\left(\beta^{\prime}+1\right)$. Now

$$
\frac{u_{2}}{u_{3}}=\sqrt{\frac{\beta \beta^{\prime}}{2 \beta(\beta+1)}}=\sqrt{\frac{\frac{3}{2} \beta+2}{2(\beta+1)}}=\frac{1}{2} \sqrt{\frac{3 \beta+4}{\beta+1}}
$$

and

$$
\frac{\tilde{\eta}}{\eta}=\frac{-2 \sqrt{\beta^{\prime}}\left(\beta^{\prime}+1\right)}{-3 \sqrt{2(\beta+1)}(\beta+2)}=\frac{2 \sqrt{\frac{3}{2} \beta+2}\left(\frac{3}{2} \beta+2+1\right)}{3 \sqrt{2(\beta+1)}(\beta+2)}=\frac{3 \sqrt{3 \beta+4}(\beta+2)}{2(3 \sqrt{\beta+1})(\beta+2)}=\frac{1}{2} \sqrt{\frac{3 \beta+4}{\beta+1}} .
$$

Since the operators $M_{1}$ and $M_{2}$ are homogeneous, combining Lemma 2.15 with Theorem 2.6 , we have the following corollary.

Corollary 2.16. For $\varphi$ in Möb, there is a unitary operator $U_{\varphi}$ such that $U_{\varphi}{ }^{-1} \tilde{\mathcal{K}}_{1}(z) U_{\varphi}=$ $\tilde{\mathcal{K}}_{2}(z)$ and $U_{\varphi}{ }^{-1}\left(\tilde{\mathcal{K}}_{1}\right)_{\bar{z}}(z) U_{\varphi}=\left(\tilde{\mathcal{K}}_{2}\right)_{\bar{z}}(z)$.

LEMma 2.17. If $\beta^{\prime}=\frac{3}{2} \beta+2$ then $\left(\tilde{\mathcal{K}}_{1}\right)_{z \bar{z}}(0)$ and $\left(\tilde{\mathcal{K}}_{2}\right)_{z \bar{z}}(0)$ are not unitarily equivalent.

Proof. By Lemma 2.12, $\left(\tilde{\mathcal{K}}_{i}\right)_{z \bar{z}}(0)=\operatorname{diag}\left(p_{i}, q_{i}, r_{i}\right)$ for $i=1,2$, where $p_{1}=\alpha, q_{1}=\alpha+$ $\beta^{\prime}\left(\beta^{\prime}+1\right), r_{1}=\alpha+\beta^{\prime}\left(-\beta^{\prime}+1\right)$ and $p_{2}=\alpha, q_{2}=\alpha+3(\beta+1)(\beta+2), r_{2}=\alpha-3 \beta(\beta+2)$. Clearly,

$$
p_{1}=p_{2}, \quad q_{1}>r_{1} \quad \text { and } q_{2}>r_{2} .
$$

If the diagonal matrices $\left(\tilde{\mathcal{K}}_{1}\right)_{z \bar{z}}(0)$ and $\left(\tilde{\mathcal{K}}_{2}\right)_{z \bar{z}}(0)$ are unitarily equivalent then $\left\{p_{1}, q_{1}, r_{1}\right\}=$ $\left\{p_{2}, q_{2}, r_{2}\right\}$, as sets. From (2.11), we see that this can happen only if $p_{1}=p_{2}, q_{1}=q_{2}$ and $r_{1}=r_{2}$. Since $\beta^{\prime}=\frac{3}{2} \beta+2, q_{1}=\alpha+\frac{3}{4}(\beta+2)(3 \beta+4)$. We see that $q_{1} \neq q_{2}$ as $\beta \neq 0$. Hence $\left(\tilde{\mathcal{K}}_{1}\right)_{z \bar{z}}(0)$ and $\left(\tilde{\mathcal{K}}_{2}\right)_{z \bar{z}}(0)$ are not unitarily equivalent.

The following theorem is now obvious.

THEOREM 2.18. The simultaneous unitary equivalence class of the curvatures and the covariant derivatives of the curvatures of order $(0,1)$ for the operators $M_{1}$ and $M_{2}$ are the same for $\beta^{\prime}=\frac{3}{2} \beta+2$. However, the covariant derivatives of the curvatures of order $(1,1)$ are not unitarily equivalent.

\section{Irreducible examples and permutation of curvature eigenvalues}

In Example 2.1, one of the two homogeneous operators $M^{*}$ is reducible while the other $M^{\prime *}$ is irreducible. Similarly in Example 2.11, one of the two operators $M_{1}^{*}$ is reducible whereas the other $M_{2}^{*}$ is irreducible. Irreducibility of $M^{\prime *}$ and $M_{2}^{*}$ follows from [16]. We are interested in constructing such examples within the class of irreducible operators in $\mathrm{B}_{n}(\mathbb{D})$. The class of irreducible homogeneous operators in $\mathrm{B}_{2}(\mathbb{D})$ cannot possibly possess such examples, since the eigenvalues of the curvature at 0 is a complete invariant for these operators (cf. $[\mathbf{1 9}]$ ). Therefore, we consider a class of homogeneous operators in $\mathrm{B}_{3}(\mathbb{D})$ discussed in $[\mathbf{1 5}]$. However, we first show that for generic bundles $E^{(\lambda, \boldsymbol{\mu})}$ the simultaneous unitary equivalence class of the curvature and the covariant derivative of the curvature of order $(0,1)$ determine the equivalence class of the homogeneous Hermitian holomorphic vector bundle $E^{(\lambda, \mu)}$. 
Notation 3.1. Let $\lambda$ be a real number and $m$ be a positive integer such that $2 \lambda-m>0$. For brevity, we will write $2 \lambda_{j}=2 \lambda-m+2 j, 0 \leqslant j \leqslant m$. Let

$$
L(\lambda)(\ell, j)= \begin{cases}\left(\begin{array}{l}
\ell \\
j
\end{array}\right)^{2} \frac{(\ell-j) !}{\left(2 \lambda_{j}\right)_{\ell-j}} & \text { for } 0 \leqslant j \leqslant \ell \leqslant m, \\
0 & \text { otherwise }\end{cases}
$$

and $\mathrm{B}=\operatorname{diag}\left(d_{0}, d_{1}, \ldots, d_{m}\right)$. Now, for $\boldsymbol{\mu}=\left(\mu_{0}, \ldots, \mu_{m}\right)^{\text {tr }}$ with $\mu_{0}=1$ and $\mu_{\ell}>0$ for $\ell=1, \ldots, m$, let

$$
\mathbf{B}^{(\lambda, \boldsymbol{\mu})}(z, w)=(1-z \bar{w})^{-2 \lambda-m} D(z \bar{w}) \exp \left(\bar{w} \mathbb{S}_{m}\right) \mathrm{B} \exp \left(z \mathbb{S}_{m}^{*}\right) D(z \bar{w}),
$$

where $B$ is a positive diagonal matrix with

$$
\mathrm{B}_{\ell, \ell}=d_{\ell}=\sum_{j=0}^{\ell}\left(\begin{array}{l}
\ell \\
j
\end{array}\right)^{2} \frac{(\ell-j) !}{\left(2 \lambda_{j}\right)_{\ell-j}} \mu_{j}^{2} \quad \text { for } 0 \leqslant \ell \leqslant m,
$$

$D(z \bar{w})=(1-z \bar{w})^{m-\ell} \delta_{p \ell}$ is diagonal and $\mathbb{S}_{m}$ is the forward shift with weight sequence $(1, \ldots, m)$. Here, $X^{\operatorname{tr}}$ denotes the transpose of the matrix $X$. Thus $L(\lambda) \boldsymbol{\mu}^{2}=\boldsymbol{d}$ for $\boldsymbol{\mu}^{2}:=\left(\mu_{0}^{2}, \mu_{1}^{2}, \ldots, \mu_{m}^{2}\right)^{\operatorname{tr}}$ and $\boldsymbol{d}=\left(d_{0}, d_{1}, \ldots, d_{m}\right)^{\operatorname{tr}}$.

The kernel $\mathbf{B}^{(\lambda, \boldsymbol{\mu})}$ is positive definite. Indeed, it is the reproducing kernel for the Hilbert space $\mathbf{A}^{(\lambda, \boldsymbol{\mu})}(\mathbb{D})$ of $\mathbb{C}^{m+1}$-valued holomorphic functions described in [15]. Let $M^{(\lambda, \boldsymbol{\mu})}$ denote the multiplication operator on the Hilbert space $\mathbf{A}^{(\lambda, \boldsymbol{\mu})}(\mathbb{D})$. The Hermitian holomorphic vector bundle associated with $\mathbf{B}^{(\lambda, \boldsymbol{\mu})}$ is denoted by $E^{(\lambda, \boldsymbol{\mu})}$. In [15], it is shown that $M^{(\lambda, \boldsymbol{\mu})}$ is an irreducible homogeneous operator, which is in $\mathrm{B}_{m+1}(\mathbb{D})$.

Lemma 3.2. For the reproducing kernel $\mathbf{B}^{(\lambda, \boldsymbol{\mu})}$, we have

(a) $\tilde{a}_{11}=\left[\mathrm{B}^{-1} \mathbb{S}_{m} \mathrm{~B}, \mathbb{S}_{m}^{*}\right]+(2 \lambda+m) I_{m+1}-2 D_{m}$,

(b) $\tilde{a}_{12}=\mathrm{B}^{1 / 2}\left(\frac{1}{2}\left(\mathrm{~B}^{-1} \mathbb{S}_{m}^{2} \mathrm{BS}_{m}^{*} \mathrm{~B}^{-1}+\mathbb{S}_{m}^{*} \mathrm{~B}^{-1} \mathbb{S}_{m}{ }^{2}\right)+\mathrm{B}^{-1}\left[D_{m}, \mathbb{S}_{m}\right]-\mathrm{B}^{-1} \mathbb{S}_{m} \mathrm{~B}_{m}^{*} \mathrm{~B}^{-1} \mathbb{S}_{m}\right) \mathrm{B}^{1 / 2}$, where $I_{k}$ denotes the identity matrix of order $k$ and $D_{m}=\operatorname{diag}(m, \ldots, 1,0)$.

Proof. From equation (2.8) in Lemma 2.12, we get $\tilde{a}_{11}=a_{00}^{-1 / 2}\left(a_{11}-a_{10} a_{00}^{-1} a_{01}\right) a_{00}^{-1 / 2}$. From the expansion of the reproducing kernel $\mathbf{B}^{(\lambda, \boldsymbol{\mu})}$ we see that $a_{00}=\mathrm{B}, a_{10}=\mathrm{BS}_{m}^{*}, a_{01}=$ $\mathbb{S}_{m} \mathrm{~B}, a_{11}=\mathbb{S}_{m} \mathrm{BS}_{m}^{*}+(2 \lambda+m) \mathrm{B}-2 D_{m} \mathrm{~B}$. Hence, $a_{11}-a_{10} a_{00}^{-1} a_{01}=\mathbb{S}_{m} \mathrm{~B}_{m}^{*}+(2 \lambda+m) \mathrm{B}-$ $2 D_{m} \mathrm{~B}-\mathrm{BS}_{m}^{*} \mathrm{~B}^{-1} \mathbb{S}_{m} \mathrm{~B}$. The proof of (a) is now complete since the matrices $\mathbb{S}_{m} \mathrm{~B} \mathbb{S}_{m}^{*}, \mathbb{S}_{m} \mathrm{~B}^{-1} \mathbb{S}_{m}^{*}$, $\mathrm{B}, \mathrm{B}^{1 / 2}, \mathrm{~B}^{-1 / 2}$ are diagonal.

From Lemma 2.5, we have $\tilde{a}_{12}=a_{00}^{1 / 2}\left(b_{00} a_{11} b_{01}+b_{00} a_{12} b_{00}-b_{10} a_{00} b_{02}\right) a_{00}^{1 / 2}$. Again, from the expansion of the reproducing kernel $\mathbf{B}^{(\lambda, \boldsymbol{\mu})}$ it is easy to see that

$$
\begin{gathered}
a_{12}=\frac{1}{2} \mathbb{S}_{m}^{2} \mathrm{~B} \mathbb{S}_{m}^{*}+(2 \lambda+m) \mathbb{S}_{m} \mathrm{~B}-D_{m} \mathbb{S}_{m} \mathrm{~B}-\mathbb{S}_{m} \mathrm{~B} D_{m}, \quad b_{00}=\mathrm{B}^{-1}, \\
b_{10}=-\mathbb{S}_{m}^{*} \mathrm{~B}^{-1}, \quad b_{02}=\frac{1}{2} \mathrm{~B}^{-1} \mathbb{S}_{m}{ }^{2} .
\end{gathered}
$$

The proof of (b) is now complete since the two diagonal matrices $\mathrm{B}$ and $D_{m}$ commute.

Let $\tilde{\mathcal{K}}^{(\lambda, \boldsymbol{\mu})}$ denote the curvature of the bundle $E^{(\lambda, \boldsymbol{\mu})}$, that is, $\tilde{\mathcal{K}}(z)=\frac{\partial}{\partial \bar{z}}\left(\tilde{h}^{-1} \frac{\partial}{\partial z} \tilde{h}\right)(z)$, where $\tilde{h}(z)=\tilde{\mathbf{B}}^{(\lambda, \boldsymbol{\mu})}(z, z)^{\text {tr }}$ for $z$ in $\mathbb{D}$. Recall that $\tilde{\mathbf{B}}^{(\lambda, \boldsymbol{\mu})}$ is the normalized reproducing kernel obtained from the reproducing $\operatorname{kernel} \mathbf{B}^{(\lambda, \boldsymbol{\mu})}$. 
LEMma 3.3. The curvature at zero $\tilde{\mathcal{K}}^{(\lambda, \mu)}(0)$ and the covariant derivative of curvature of order $(0,1)$ at zero $\left(\tilde{\mathcal{K}}^{(\lambda, \mu)}\right)_{\bar{z}}(0)$ are given by the formulae

(a) $\tilde{\mathcal{K}}^{(\lambda, \boldsymbol{\mu})}(0)=\operatorname{diag}\left(\left(2 \lambda_{r}+\alpha_{r}-\alpha_{r+1}\right)_{r=0}^{m}\right)$,

(b) $\left(\tilde{\mathcal{K}}^{(\lambda, \mu)}\right)_{\bar{z}}(0)=2 S\left(\left(-\sqrt{\alpha_{r}}\left(1+\alpha_{r}-\frac{1}{2}\left(\alpha_{r-1}+\alpha_{r+1}\right)\right)_{r=1}^{m}\right)^{\text {tr }}\right.$, where $\alpha_{r}=r^{2} d_{r-1} d_{r}^{-1}$ for $0 \leqslant r \leqslant m$ with $\alpha_{0}=\alpha_{m+1}=0$.

Proof. We only write the non-zero entries of the matrices involved. Notice that $\mathbb{S}_{m} \mathrm{BS}_{m}^{*}(r, r)=r^{2} d_{r-1}$ for $1 \leqslant r \leqslant m, \quad \mathrm{~B}^{-1} \mathbb{S}_{m} \mathrm{BS}_{m}^{*}(r, r)=r^{2} d_{r-1} d_{r}^{-1}$ for $1 \leqslant r \leqslant m$, $\mathbb{S}_{m}^{*} \mathrm{~B}^{-1} \mathbb{S}_{m}(r, r)=(r+1)^{2} d_{r+1}^{-1}$ for $0 \leqslant r \leqslant m-1$ and $\mathbb{S}_{m}^{*} \mathrm{~B}^{-1} \mathbb{S}_{m} \mathrm{~B}(r, r)=(r+1)^{2} d_{r} d_{r+1}^{-1}$ for $0 \leqslant r \leqslant m-1$. Therefore, by Lemma 3.2(a), we see that $\tilde{\mathcal{K}}^{(\lambda, \boldsymbol{\mu})}(0)=\tilde{a}_{11}^{\mathrm{tr}}=\operatorname{diag}\left(\left\{2 \lambda_{r}+\alpha_{r}-\right.\right.$ $\left.\left.\alpha_{r+1}\right\}_{r=0}^{m}\right)$. This proves part (a).

To prove part (b), we observe that

$$
\begin{aligned}
\mathrm{B}_{m}(r+1, r) & =(r+1) d_{r+1}^{-1} \quad \text { for } 0 \leqslant r \leqslant m-1, \\
\mathbb{S}_{m} \mathrm{BS}_{m}^{*}(r, r) & =r^{2} d_{r-1} \quad \text { for } 1 \leqslant r \leqslant m \\
\mathrm{~B}^{-1} \mathbb{S}_{m}^{2} \mathrm{BS}_{m}^{*} \mathrm{~B}^{-1}(r+1, r) & =r^{2}(r+1) d_{r-1} d_{r}^{-1} d_{r+1}^{-1} \quad \text { for } 1 \leqslant r \leqslant m-1 .
\end{aligned}
$$

Equivalently,

$$
\mathrm{B}^{-1} \mathbb{S}_{m}^{2} \mathrm{BS}_{m}^{*} \operatorname{tr} B^{-1}(r, r-1)=r(r-1)^{2} d_{r-2} d_{r-1}^{-1} d_{r}^{-1} \quad \text { for } 2 \leqslant r \leqslant m .
$$

Since

$$
\begin{aligned}
\mathbb{S}_{m}^{*} \mathrm{~B}^{-1} \mathbb{S}_{m}^{2}(r, r-1) & =r(r+1)^{2} d_{r+1}^{-1} \quad \text { for } 1 \leqslant r \leqslant m-1, \\
D_{m} \mathbb{S}_{m}(r, r-1) & =(m-r) r \quad \text { for } 1 \leqslant r \leqslant m, \\
\mathbb{S}_{m} D_{m}(r, r-1) & =r(m-r+1) \quad \text { for } 1 \leqslant r \leqslant m,
\end{aligned}
$$

it follows that

$$
\left[D_{m}, \mathbb{S}_{m}\right](r, r-1)=-r, \quad \text { that is, }\left[D_{m}, \mathbb{S}_{m}\right]=-\mathbb{S}_{m} .
$$

Hence $\left(\mathrm{B}^{-1}\left[D_{m}, \mathbb{S}_{m}\right]\right)(r, r-1)=-r d_{r}^{-1}$ for $1 \leqslant r \leqslant m$. Now,

$$
\begin{aligned}
\left(\mathrm{B}^{-1}\left[D_{m}, \mathbb{S}_{m}\right]-\mathrm{B}^{-1} \mathbb{S}_{m} \mathrm{BS}_{m}^{*} \mathrm{~B}^{-1} \mathbb{S}_{m}\right)(r, r-1) & =-r d_{r}^{-1}-r^{3} d_{r-1} d_{r}^{-2} \\
& =-r d_{r}^{-1}\left(1+r^{2} d_{r-1} d_{r}^{-1}\right) \\
& =-r d_{r}^{-1}\left(1+\alpha_{r}\right) \quad \text { for } 1 \leqslant r \leqslant m .
\end{aligned}
$$

Also,

$$
\begin{aligned}
\frac{1}{2}\left(\mathrm{~B}^{-1} \mathbb{S}_{m}^{2} \mathrm{BS}_{m}^{*} \mathrm{~B}^{-1}+\mathbb{S}_{m}^{*} \mathrm{~B}^{-1} \mathbb{S}_{m}^{2}\right)(r, r-1) & =\frac{r}{2}\left((r-1)^{2} d_{i-2} d_{r-1}^{-1} d_{r}^{-1}+(r+1)^{2} d_{r+1}^{-1}\right) \\
& =\frac{r}{2}\left(\alpha_{r-1} d_{r}^{-1}+(r+1)^{2} d_{r+1}^{-1}\right)
\end{aligned}
$$

for $1 \leqslant r \leqslant m$ with $\alpha_{0}=0=d_{m+1}^{-1}$. From Lemma 3.2(b), using $d_{-1}=0=d_{m+1}^{-1}$ we get

$$
\begin{aligned}
\tilde{a}_{12}(r, r-1) & =\frac{r}{2}\left(d_{r-1} d_{r}\right)^{1 / 2}\left(\alpha_{r-1} d_{r}^{-1}+(r+1)^{2} d_{r+1}^{-1}\right)-r\left(d_{r-1} d_{r}\right)^{1 / 2} d_{r}^{-1}\left(1+\alpha_{r}\right) \\
& =\frac{r}{2}\left(d_{r-1} d_{r}^{-1}\right)^{1 / 2}\left(\alpha_{r-1}+(r+1)^{2} d_{r} d_{r+1}^{-1}\right)-r\left(d_{r-1} d_{r}^{-1}\right)^{1 / 2}\left(1+\alpha_{r}\right) \\
& =-\sqrt{\alpha_{r}}\left(1+\alpha_{r}-\frac{1}{2}\left(\alpha_{r-1}+\alpha_{r+1}\right)\right)
\end{aligned}
$$

for $1 \leqslant r \leqslant m$. This proves part (b). 
Notation 3.4. Let $\delta_{r+1}=2 \lambda_{r}+\alpha_{r}-\alpha_{r+1}$ for $0 \leqslant r \leqslant m$ and let $\theta_{\ell}=-\sqrt{\alpha_{\ell}}(1+$ $\left.\alpha_{\ell}-\frac{1}{2}\left(\alpha_{\ell-1}+\alpha_{\ell+1}\right)\right)$ for $1 \leqslant \ell \leqslant m$. In this notation, $\tilde{\mathcal{K}}^{(\lambda, \mu)}(0)=\operatorname{diag}\left(\left(\delta_{r+1}\right)_{r=0}^{m}\right)$ and $\left(\tilde{\mathcal{K}}^{(\lambda, \mu)}\right)_{\bar{z}}(0)=2 S\left(\left(\theta_{\ell}\right)_{\ell=1}^{m}\right)^{\text {tr }}$. As in the previous lemma, we will let $\alpha_{r}=r^{2} d_{r-1} d_{r}^{-1}$ for $0 \leqslant$ $r \leqslant m$ with $\alpha_{0}=\alpha_{m+1}=0$.

REMARK 3.5. We emphasize that the reproducing kernel $\mathbf{B}^{(\lambda, \boldsymbol{\mu})}$ is computed using an ordered basis, that is, $\mathbf{B}^{(\lambda, \boldsymbol{\mu})}(w, w)=\left(\left(\left\langle\gamma_{i}(w), \gamma_{j}(w)\right\rangle\right)\right)_{i, j=1}^{m+1}$, where $\left\{\gamma_{i}(w)\right\}_{i=1}^{m+1}$ is an ordered basis. Consequently, the set of eigenvalues of $\tilde{\mathcal{K}}^{(\lambda, \mu)}(0)$, which is diagonal, appear in a fixed order. If one considers $\left\{\gamma_{\sigma(i)}(w)\right\}_{i=1}^{m+1}$, it will give rise to a different reproducing kernel $\quad P_{\sigma} \mathbf{B}^{(\lambda, \boldsymbol{\mu})} P_{\sigma}^{*}$, say $\mathbf{B}_{\sigma}^{(\lambda, \boldsymbol{\mu})}$, where $\sigma \in S_{m+1}, S_{m+1}$ denotes the symmetric group of degree $(m+1)$ and $P_{\sigma}(i, j)=\delta_{\sigma(i), j}$. Hence $\tilde{\mathcal{K}}_{\sigma}(0)=\operatorname{diag}\left(\left(\delta_{\sigma(r+1)}\right)_{r=0}^{m}\right)$, where $\tilde{\mathcal{K}}_{\sigma}$ is the curvature with respect to the metric $\tilde{h}_{\sigma}(z)=\tilde{\mathbf{B}}_{\sigma}^{(\lambda, \boldsymbol{\mu})}(z, z)^{\mathrm{tr}}$. It follows that the curvature of the corresponding bundle as a matrix depends on the choice of the particular ordered basis. The set of eigenvalues of curvature at 0 , which is diagonal in our case, will be thought of as an ordered tuple, namely, the ordered set of diagonal elements of $\tilde{\mathcal{K}}^{(\lambda, \boldsymbol{\mu})}(0)$.

Definition 3.6 ([7, Def. 3.18, p. 226]). A $C^{\infty}$ vector bundle $E$ over an open subset $\Omega$ of $\mathbb{C}$ with metric-preserving connection $D$ is said to be generic if $\mathcal{K}$ has distinct eigenvalues of multiplicity one at each point of $\Omega$.

From Lemma 2.8(a) and Lemma 3.3(a), we note that $E^{(\lambda, \boldsymbol{\mu})}$ is generic if and only if $\delta_{r+1}$ are all distinct for $0 \leqslant r \leqslant m$. Thus the proof of the following corollary is complete.

Corollary 3.7. We have $\delta_{r}=\delta_{r+1}$ if and only if $\theta_{r}=0$ for $1 \leqslant r \leqslant m$ with $\alpha_{0}=\alpha_{m+1}=$ 0 . In particular, if $E^{(\lambda, \boldsymbol{\mu})}$ is generic then $\theta_{r} \neq 0$ for $1 \leqslant r \leqslant m$.

LEMMA 3.8. If $\left(\delta_{r+1}\right)_{r=0}^{m}$ is an ordered tuple of positive numbers such that $\tilde{\mathcal{K}}^{(\lambda, \boldsymbol{\mu})}(0)=$ $\operatorname{diag}\left(\left(\delta_{r+1}\right)_{r=0}^{m}\right)$, then we have:

(i) $\sum_{k=0}^{m} \delta_{k+1}>m(m+1)$;

(ii) $r /(m+1) \sum_{k=0}^{m} \delta_{k+1}-\sum_{k=0}^{r-1} \delta_{k+1}>r(m+1-r)$ for $1 \leqslant r \leqslant m$.

Proof. By Lemma 3.3, Remark 3.5 and the hypothesis of the lemma, we have

$$
2 \lambda_{r}+\alpha_{r}-\alpha_{r+1}=\delta_{r+1}
$$

for $0 \leqslant r \leqslant m$. This is the same as $A \boldsymbol{x}=\boldsymbol{b}$, where

$$
A(i, j)=\left\{\begin{aligned}
-1, & j=i+1 \\
1, & j=0 \text { or } i=j \\
0, & \text { otherwise }
\end{aligned}\right.
$$

for $0 \leqslant i, j \leqslant m ; \boldsymbol{x}(0)=2 \lambda_{0}, \boldsymbol{x}(r)=\alpha_{r}$ and $\boldsymbol{b}(r)=\delta_{r+1}-2 r$ for $0 \leqslant r \leqslant m$. 
We observe that $\operatorname{det} A=\left(\begin{array}{cc}m+1 & 0 \\ B & A^{\prime}\end{array}\right)=m+1$, where $B(i)=1$ for $1 \leqslant i \leqslant m$ and $A^{\prime}$ is an upper-triangular matrix of size $m$ with 1 as its diagonal entries. Thus, the system $A \boldsymbol{x}=\boldsymbol{b}$ of linear equations admits a unique solution. One verifies that

$2 \lambda_{0}=\frac{1}{m+1} \sum_{k=0}^{m} \delta_{k+1}-m \quad$ and $\alpha_{r}=\frac{r}{m+1} \sum_{k=0}^{m} \delta_{k+1}-\sum_{k=0}^{r-1} \delta_{k+1}-r(m+1-r) \quad$ for $1 \leqslant r \leqslant m$.

Recall that $2 \lambda_{0}$ and $\alpha_{r}=r^{2} d_{r-1} d_{r}^{-1}$ (for $1 \leqslant r \leqslant m$ ) are all positive. Therefore, a set of necessary conditions for existence of the positive numbers $\left\{\delta_{r+1}\right\}_{r=0}^{m}$ such that $\tilde{\mathcal{K}}^{(\lambda, \boldsymbol{\mu})}(0)=$ $\operatorname{diag}\left(\left(\delta_{r+1}\right)_{r=0}^{m}\right)$ are the inequalities in the statement of the lemma.

As described in Notation 3.1, let $\boldsymbol{\mu}=\left(1, \mu_{1}, \ldots, \mu_{m}\right)^{\operatorname{tr}}$ and $\boldsymbol{\mu}^{\prime}=\left(1, \mu_{1}^{\prime}, \ldots, \mu_{m}^{\prime}\right)^{\text {tr }}$ with $\mu_{\ell}, \mu_{\ell}^{\prime}>0$ for $0 \leqslant \ell \leqslant m ; \boldsymbol{\alpha}=\left(\alpha_{1}, \ldots, \alpha_{m}\right)$ and $\boldsymbol{\alpha}^{\prime}=\left(\alpha_{1}^{\prime}, \ldots, \alpha_{m}^{\prime}\right)$. For $0 \leqslant j \leqslant m$, set $2 \gamma_{j}=$ $2 \gamma-m+2 j$, where $\gamma=\lambda$ or $\gamma=\lambda^{\prime}$. Set $\boldsymbol{d}=L(\lambda) \boldsymbol{\mu}^{2}, \boldsymbol{d}^{\prime}=L\left(\lambda^{\prime}\right) \boldsymbol{\mu}^{\prime 2}$, where $\boldsymbol{\mu}^{2}$ and $\boldsymbol{\mu}^{\prime 2}$ denote the componentwise square of $\boldsymbol{\mu}$ and $\boldsymbol{\mu}^{\prime}$. Let $2 \lambda_{0}=2 \lambda-m, \alpha_{i}=i^{2} d_{i-1} d_{i}{ }^{-1} ; 2 \lambda_{0}^{\prime}=2 \lambda^{\prime}-m$, $\alpha_{i}^{\prime}=i^{2} d_{i-1}^{\prime} d_{i}{ }^{-1}$ for $0 \leqslant i \leqslant m$. In this notation, we have the following.

Lemma 3.9. We have $\left(\begin{array}{c}\lambda \\ \boldsymbol{\mu}\end{array}\right)=\left(\begin{array}{c}\lambda^{\prime} \\ \boldsymbol{\mu}^{\prime}\end{array}\right)$ if and only if $\left(2 \lambda_{0}, \boldsymbol{\alpha}\right)=\left(2 \lambda_{0}^{\prime}, \boldsymbol{\alpha}^{\prime}\right)$.

Proof. We prove the 'only if' part. Assuming $\left(2 \lambda_{0}, \boldsymbol{\alpha}\right)=\left(2 \lambda_{0}^{\prime}, \boldsymbol{\alpha}^{\prime}\right)$ we have $\lambda=\lambda^{\prime}$ and $\alpha_{i}=\alpha_{i}^{\prime}$ for $1 \leqslant i \leqslant m$. Thus $\boldsymbol{d}=\boldsymbol{d}^{\prime}$. Now invertibility of $L(\lambda)$ implies that $\boldsymbol{\mu}^{2}=\boldsymbol{\mu}^{\prime 2}$, that is, $\boldsymbol{\mu}=\boldsymbol{\mu}^{\prime}$.

Corollary 3.10. Suppose that $\mathbf{B}^{(\lambda, \boldsymbol{\mu})}$ and $\mathbf{B}^{\left(\lambda^{\prime}, \boldsymbol{\mu}^{\prime}\right)}$ are such that $\tilde{\mathcal{K}}^{(\lambda, \boldsymbol{\mu})}(0)=\tilde{\mathcal{K}}^{\left(\lambda^{\prime}, \boldsymbol{\mu}^{\prime}\right)}(0)$. Then $\left(\begin{array}{l}\lambda \\ \boldsymbol{\mu}\end{array}\right)=\left(\begin{array}{c}\lambda^{\prime} \\ \boldsymbol{\mu}^{\prime}\end{array}\right)$.

Proof. Let $\tilde{\mathcal{K}}^{(\lambda, \boldsymbol{\mu})}(0)=\tilde{\mathcal{K}}^{\left(\lambda^{\prime}, \boldsymbol{\mu}^{\prime}\right)}(0)=\operatorname{diag}\left(\left(\delta_{r+1}\right)_{r=0}^{m}\right)$. Consider the system of linear equations $A \boldsymbol{x}=\boldsymbol{b}$ and $A \boldsymbol{x}^{\prime}=\boldsymbol{b}$, where $A, \boldsymbol{x}, \boldsymbol{b}$ are as in Lemma 3.9 and $\boldsymbol{x}^{\prime}(0)=2 \lambda_{0}^{\prime}, \boldsymbol{x}^{\prime}(r)=\alpha_{r}^{\prime}$ for $1 \leqslant r \leqslant m$. Since $\operatorname{det} A=m+1, A$ is invertible. Hence $\boldsymbol{x}=\boldsymbol{x}^{\prime}$, that is, $\left(2 \lambda_{0}, \boldsymbol{\alpha}\right)=\left(2 \lambda_{0}^{\prime}, \boldsymbol{\alpha}^{\prime}\right)$, where $\boldsymbol{\alpha}, \boldsymbol{\alpha}^{\prime}$ are as in Lemma 3.9. Now by Lemma 3.9, we have $\left(\begin{array}{l}\lambda \\ \boldsymbol{\mu}\end{array}\right)=\left(\begin{array}{l}\lambda^{\prime} \\ \boldsymbol{\mu}^{\prime}\end{array}\right)$.

Recall that $M^{(\lambda, \boldsymbol{\mu})}$ and $E^{(\lambda, \boldsymbol{\mu})}$ denote the multiplication operator and Hermitian holomorphic vector bundle associated with the reproducing kernel $\mathbf{B}^{(\lambda, \boldsymbol{\mu})}$, respectively. We recall a theorem from $[\mathbf{1 5}]$.

Theorem 3.11 ([15, Theorem 6.2]). The reproducing kernels $\mathbf{B}^{(\lambda, \boldsymbol{\mu})}$ and $\mathbf{B}^{\left(\lambda^{\prime}, \boldsymbol{\mu}^{\prime}\right)}$ are equivalent; that is, the multiplication operators $M^{(\lambda, \boldsymbol{\mu})}$ and $M^{\left(\lambda^{\prime}, \boldsymbol{\mu}^{\prime}\right)}$ are unitarily equivalent if and only if $\left(\begin{array}{l}\lambda \\ \boldsymbol{\mu}\end{array}\right)=\left(\begin{array}{c}\lambda^{\prime} \\ \boldsymbol{\mu}^{\prime}\end{array}\right)$.

The following corollary is an easy consequence of Corollary 3.10 and Theorem 3.11.

Corollary 3.12. Suppose the $\mathbf{B}^{(\lambda, \boldsymbol{\mu})}$ and $\mathbf{B}^{\left(\lambda^{\prime}, \boldsymbol{\mu}^{\prime}\right)}$ are such that $\tilde{\mathcal{K}}^{(\lambda, \boldsymbol{\mu})}(0)=\tilde{\mathcal{K}}^{\left(\lambda^{\prime}, \boldsymbol{\mu}^{\prime}\right)}(0)$. Then the multiplication operators $M^{(\lambda, \mu)}$ and $M^{\left(\lambda^{\prime}, \boldsymbol{\mu}^{\prime}\right)}$ are unitarily equivalent. 
Now we state the main theorem of this section.

TheOREM 3.13. Suppose that the Hermitian holomorphic vector bundles $E^{(\lambda, \mu)}$ and $E^{\left(\lambda^{\prime}, \boldsymbol{\eta}\right)}$ are generic. Then the multiplication operators $M^{(\lambda, \boldsymbol{\mu})}$ and $M^{\left(\lambda^{\prime}, \boldsymbol{\eta}\right)}$ are unitarily equivalent if $\tilde{\mathcal{K}}^{(\lambda, \boldsymbol{\mu})}(0)$ and $\left(\tilde{\mathcal{K}}^{(\lambda, \boldsymbol{\mu})}\right)_{\bar{z}}(0)$ are simultaneously unitarily equivalent to $\tilde{\mathcal{K}}^{\left(\lambda^{\prime}, \boldsymbol{\eta}\right)}(0)$ and $\left(\tilde{\mathcal{K}}^{\left(\lambda^{\prime}, \boldsymbol{\eta}\right)}\right)_{\bar{z}}(0)$, respectively.

The proof of this theorem will be complete after proving a sequence of lemmas. We omit the easy proof of the first of these lemmas.

Lemma 3.14. Suppose that $\Delta=\left(\left(k_{i} \delta_{i j}\right)_{i, j=1}^{n}, \Delta_{\sigma}=\left(\left(k_{\sigma(i)} \delta_{i j}\right)\right)_{i, j=1}^{n}, k_{i} \neq k_{j}\right.$ if $i \neq j$ and $C$ in $\mathcal{M}_{n}$ is such that $C \Delta=\Delta_{\sigma} C$. Then $C=\left(\left(C_{i j} \delta_{\sigma(i), j}\right)\right)_{i, j=1}^{n}$ for $C_{i j} \in \mathbb{C}$ and $i, j=1, \ldots, n$, where $\sigma$ is in $S_{n}$, and $S_{n}$ denotes the permutation group of degree $n$.

Lemma 3.15. Suppose that $B$ in $\mathcal{M}_{n+1}$ is such that $B S\left(\left(\beta_{k}\right)_{k=1}^{n}\right)^{\operatorname{tr}}=S\left(\left(\beta_{k}\right)_{k=1}^{n}\right)^{\operatorname{tr}} B$ for $\beta_{k} \neq 0,1 \leqslant k \leqslant n$. Then $B$ is upper-triangular.

Proof. Let $B=((B(i, j)))_{i, j=1}^{n+1}$. The $(i, 1)$ th entries of $B S\left(\left(\beta_{k}\right)_{k=1}^{n}\right)^{\operatorname{tr}}$ and $S\left(\left(\beta_{k}\right)_{k=1}^{n}\right)^{\operatorname{tr}} B$ are 0 and $\beta_{i} B(i+1,1)$ for $1 \leqslant i \leqslant n$, respectively. By hypothesis, $B(i+1,1)=0$ for $1 \leqslant i \leqslant n$. We want to show that $B(i+1, j)=0$ for $j \leqslant i \leqslant n, 1 \leqslant j \leqslant n$. We prove this by induction. We know that the assertion is true for $j=1$. Assume that $B(i+1, j-1)=0$ for $j-1 \leqslant$ $i \leqslant n, 2 \leqslant j \leqslant n+1$; equivalently, $B(i, j-1)=0$ for $j \leqslant i \leqslant n+1,2 \leqslant j \leqslant n+1$. Equating $(i, j)$ th entries from $B S\left(\left(\beta_{k}\right)_{k=1}^{n}\right)^{\operatorname{tr}}$ and $S\left(\left(\beta_{k}\right)_{k=1}^{n}\right)^{\operatorname{tr}} B$ we have

$$
B(i, j-1) \beta_{j-1}=\beta_{i} B(i+1, j) \text { for } 1 \leqslant i \leqslant n, 2 \leqslant j \leqslant n+1 .
$$

We note that the left-hand side of the above equality is zero for $j \leqslant i \leqslant n+1,2 \leqslant j \leqslant n+1$, by induction hypothesis. Hence $B(i+1, j)=0$ for $j \leqslant i \leqslant n+1,2 \leqslant j \leqslant n+1$ as $\beta_{i} \neq 0$ for $j \leqslant i \leqslant n+1$.

Lemma 3.16. Suppose that $C=\left(\left(C_{i j} \delta_{\sigma(i), j}\right)\right)_{i, j=1}^{n}$ for $C_{i j} \in \mathbb{C}, i, j=1, \ldots, n$ and $\sigma$ is in $S_{n}$, where $S_{n}$ denotes the permutation group of degree $n$. Then $|\operatorname{det} C|=\prod_{i=1}^{n}\left|C_{i, \sigma(i)}\right|$.

Proof. We observe that the only possible non-zero entries of $C$ are the $(i, \sigma(i))$ th entries for $1 \leqslant i \leqslant n+1$ and $C(i, \sigma(i))=C_{i, \sigma(i)}$. Let $\tilde{C}=\operatorname{diag}\left(\left(C_{i, \sigma(i)}\right)_{i=1}^{n+1}\right)$. It is easy to see that $|\operatorname{det} \tilde{C}|=|\operatorname{det} C|$, as $\tilde{C}$ can be converted to $C$ by interchanging its rows and columns. This proves the lemma.

The next corollary is immediate.

Corollary 3.17. If $C=\left(\left(C_{i j} \delta_{\sigma(i), j}\right)\right)_{i, j=1}^{n}$ then $C$ is invertible if and only if $C_{i, \sigma(i)} \neq 0$ for $\sigma \in S_{n}, 1 \leqslant i \leqslant n+1$, where $S_{n}$ denotes the permutation group of degree $n$.

Lemma 3.18. If $C$ is invertible and satisfies the hypothesis of Lemma 3.14 for id $\neq \sigma \in S_{n}$ then $C$ cannot be a triangular matrix. 
Proof. From Lemma 3.14 and Corollary 3.17, it follows that the only non-zero entries of $C$ are the $(i, \sigma(i))$ th entries for $1 \leqslant i \leqslant n+1$ and $C(i, \sigma(i))=C_{i, \sigma(i)}$. Therefore, it suffices to show that there exist $1 \leqslant i, j \leqslant n$ with $i \neq j$ such that $i>\sigma(i)$ and $j<\sigma(j)$ for id $\neq \sigma \in S_{n}$. Since $\sigma \neq \mathrm{id}$, there is an $i, 1 \leqslant i \leqslant n$, such that $\sigma(i) \neq i$. Without loss of generality assume that $i>\sigma(i)$. Now, if possible, let $r \geqslant \sigma(r)$ for $1 \leqslant r \leqslant n$ with strict inequalities for some $r$. Since $\sigma$ is a one-to-one map of the finite set $\{1, \ldots, n\}$ onto itself, this is not possible by the pigeon hole principle. Hence there is a $j, 1 \leqslant j \leqslant n$, such that $j<\sigma(j)$.

Proof of Theorem 3.13. By hypothesis there is an $L \in G L(m+1, \mathbb{C})$ such that:

(i) $L^{-1} \tilde{\mathcal{K}}^{(\lambda, \boldsymbol{\mu})}(0) L=\tilde{\mathcal{K}}^{\left(\lambda^{\prime}, \boldsymbol{\eta}\right)}(0)$;

(ii) $L^{-1}\left(\tilde{\mathcal{K}}^{(\lambda, \boldsymbol{\mu})}\right)_{\bar{z}}(0) L=\left(\tilde{\mathcal{K}}^{\left(\lambda^{\prime}, \boldsymbol{\eta}\right)}\right)_{\bar{z}}(0)$.

Clearly (i) implies that the sets of eigenvalues of $\tilde{\mathcal{K}}^{(\lambda, \boldsymbol{\mu})}(0)$ and $\tilde{\mathcal{K}}^{\left(\lambda^{\prime}, \boldsymbol{\eta}\right)}(0)$ are the same. Since $\tilde{\mathcal{K}}^{(\lambda, \boldsymbol{\mu})}(0)$ and $\tilde{\mathcal{K}}^{\left(\lambda^{\prime}, \boldsymbol{\eta}\right)}(0)$ are diagonal matrices it follows that either

(a) $\tilde{\mathcal{K}}^{(\lambda, \boldsymbol{\mu})}(0)=\tilde{\mathcal{K}}^{\left(\lambda^{\prime}, \boldsymbol{\eta}\right)}(0)$, or

(b) the set of diagonal entries of $\tilde{\mathcal{K}}^{(\lambda, \boldsymbol{\mu})}(0)$ equals the set of diagonal entries of $\tilde{\mathcal{K}}^{\left(\lambda^{\prime}, \boldsymbol{\eta}\right)}(0)$ but $\tilde{\mathcal{K}}^{(\lambda, \boldsymbol{\mu})}(0) \neq \tilde{\mathcal{K}}^{\left(\lambda^{\prime}, \boldsymbol{\eta}\right)}(0)$.

Now, (b) is equivalent to the statement that $\tilde{\mathcal{K}}^{\left(\lambda^{\prime}, \boldsymbol{\eta}\right)}(0)=\operatorname{diag}\left(\left(\delta_{\sigma(r+1)}\right)_{r=0}^{m}\right)$ for id $\neq \sigma \in S_{m+1}$, where $\left.\tilde{\mathcal{K}}^{(\lambda, \boldsymbol{\mu})}(0)=\operatorname{diag}\left(\left(\delta_{r+1}\right)_{r=0}^{m}\right)\right)$. This implies by Lemma 3.18 that $L$ cannot be a triangular matrix. Whereas (ii) implies by Corollary 3.7 and Lemma 3.15 that $L$ is an upper-triangular matrix. Hence (b) and (ii) cannot occur simultaneously. Having ruled out the possibility of (b), we conclude that (a) must occur. Therefore, by Corollary 3.12, we have $\left(\begin{array}{c}\lambda \\ \mu\end{array}\right)=\left(\begin{array}{c}\lambda^{\prime} \\ \eta\end{array}\right)$.

\section{Homogeneous bundles of rank 3}

Now we specialize to the case $m=2$. In this case, conclusions similar to those of Theorem 3.13 are true even if $E^{(\lambda, \mu)}$ is not assumed to be generic. Recall that the rank of the bundle $E^{(\lambda, \mu)}$ is 3 when $m=2$.

Theorem 4.1. For $m=2$, the multiplication operators $M^{(\lambda, \boldsymbol{\mu})}$ and $M^{\left(\lambda^{\prime}, \boldsymbol{\eta}\right)}$ are unitarily equivalent if $\tilde{\mathcal{K}}^{(\lambda, \boldsymbol{\mu})}(0)$ and $\left(\tilde{\mathcal{K}}^{(\lambda, \boldsymbol{\mu})}\right)_{\bar{z}}(0)$ are simultaneously unitarily equivalent to $\tilde{\mathcal{K}}^{\left(\lambda^{\prime}, \boldsymbol{\eta}\right)}(0)$ and $\left(\tilde{\mathcal{K}}^{\left(\lambda^{\prime}, \boldsymbol{\eta}\right)}\right)_{\bar{z}}(0)$, respectively.

Proof. By Theorem 3.13, we only need to consider the case when one of $E^{(\lambda, \boldsymbol{\mu})}$ and $E^{\left(\lambda^{\prime}, \boldsymbol{\eta}\right)}$ is not generic.

Let $\tilde{\mathcal{K}}^{(\lambda, \boldsymbol{\mu})}=\operatorname{diag}\left(\delta_{1}, \delta_{2}, \delta_{3}\right)$ and $\tilde{\mathcal{K}}^{\left(\lambda^{\prime}, \boldsymbol{\eta}\right)}=\operatorname{diag}\left(\delta_{1}^{\prime}, \delta_{2}^{\prime}, \delta_{3}^{\prime}\right)$, where $\delta_{i+1}=2 \lambda_{i}+\alpha_{i}-\alpha_{i+1}$, $\delta_{i+1}^{\prime}=2 \lambda_{i}^{\prime}+\alpha_{i}^{\prime}-\alpha_{i+1}^{\prime} \quad$ with $2 \lambda_{i}=2 \lambda-2+2 i, \quad 2 \lambda_{i}^{\prime}=2 \lambda^{\prime}-2+2 i, \quad \alpha_{i}=i^{2} d_{i-1} d_{i}^{-1}, \quad \alpha_{i}^{\prime}=$ $i^{2} d_{i-1}^{\prime} d_{i}^{\prime-1}$ for $i=0,1,2 ; \alpha_{0}=\alpha_{3}=\alpha_{0}^{\prime}=\alpha_{3}^{\prime}=0$ and $\mathbf{B}^{(\lambda, \boldsymbol{\mu})}(0,0)=\operatorname{diag}\left(d_{0}, d_{1}, d_{2}\right), d_{0}=1$; $\mathbf{B}^{\left(\lambda^{\prime}, \boldsymbol{\eta}\right)}(0,0)=\operatorname{diag}\left(d_{0}^{\prime}, d_{1}^{\prime}, d_{2}^{\prime}\right), d_{0}^{\prime}=1$. We observe that $\delta_{3}-\delta_{1}=\alpha_{1}+\alpha_{2}+4>0$ and $\delta_{3}^{\prime}-$ $\delta_{1}^{\prime}=\alpha_{1}^{\prime}+\alpha_{2}^{\prime}+4>0$. Now assume that

(i) $L^{-1} \tilde{\mathcal{K}}^{(\lambda, \boldsymbol{\mu})}(0) L=\tilde{\mathcal{K}}^{\left(\lambda^{\prime}, \boldsymbol{\eta}\right)}(0)$ for some $L \in G L(3, \mathbb{C})$.

It follows easily from (i) that if one of the two bundles is not generic then the other cannot be generic. Noting that $\tilde{\mathcal{K}}^{(\lambda, \boldsymbol{\mu})}(0)$ and $\tilde{\mathcal{K}}^{\left(\lambda^{\prime}, \boldsymbol{\eta}\right)}(0)$ are diagonal matrices, we have the following possibilities.
(a) $\delta_{1}=\delta_{2}$ and $\delta_{1}^{\prime}=\delta_{2}^{\prime}$,
(b) $\delta_{2}=\delta_{3}$ and $\delta_{2}^{\prime}=\delta_{3}^{\prime}$,
(c) $\delta_{1}=\delta_{2}$ and $\delta_{2}^{\prime}=\delta_{3}^{\prime}$,
(d) $\delta_{2}=\delta_{3}$ and $\delta_{1}^{\prime}=\delta_{2}^{\prime}$.

From (a) we have $\delta_{1}=\delta_{2}<\delta_{3}$ and $\delta_{1}^{\prime}=\delta_{2}^{\prime}<\delta_{3}^{\prime}$. Since (i) implies that $\left\{\delta_{1}, \delta_{2}, \delta_{3}\right\}=$ $\left\{\delta_{1}^{\prime}, \delta_{2}^{\prime}, \delta_{3}^{\prime}\right\}$, comparing the order of magnitude we see that $\delta_{1}=\delta_{1}^{\prime}, \delta_{2}=\delta_{2}^{\prime}$ and $\delta_{3}=\delta_{3}^{\prime}$. Hence 
$\tilde{\mathcal{K}}^{(\lambda, \boldsymbol{\mu})}=\tilde{\mathcal{K}}^{\left(\lambda^{\prime}, \boldsymbol{\eta}\right)}$. Therefore by Corollary 3.12 , we have $\left(\begin{array}{c}\lambda \\ \boldsymbol{\mu}\end{array}\right)=\left(\begin{array}{c}\lambda^{\prime} \\ \boldsymbol{\eta}\end{array}\right)$. Thus, $M^{(\lambda, \boldsymbol{\mu})}$ and $M^{\left(\lambda^{\prime}, \boldsymbol{\eta}\right)}$ are unitarily equivalent.

A similar argument shows that the assumptions in (b) lead to the same conclusion.

From (c), we have $\delta_{1}=\delta_{2}<\delta_{3}$ and $\delta_{1}^{\prime}<\delta_{2}^{\prime}=\delta_{3}^{\prime}$. From (i) we have $\left\{\delta_{1}, \delta_{2}, \delta_{3}\right\}=\left\{\delta_{1}^{\prime}, \delta_{2}^{\prime}, \delta_{3}^{\prime}\right\}$, as sets. Comparing the order of magnitude we get $\delta_{1}=\delta_{2}=\delta_{1}^{\prime}$ and $\delta_{3}=\delta_{2}^{\prime}=\delta_{3}^{\prime}$. Comparing multiplicities of $\delta_{1}$ and $\delta_{2}^{\prime}$ we have $\delta_{1}=\delta_{2}^{\prime}$ and $\delta_{3}=\delta_{1}^{\prime}$. All the equalities together imply that $\delta_{1}=\delta_{3}$ and $\delta_{1}^{\prime}=\delta_{3}^{\prime}$, which is impossible. Similarly we see that (d) is also impossible as $\delta_{3}>\delta_{1}$ and $\delta_{3}^{\prime}>\delta_{1}^{\prime}$. This completes the proof.

If $\delta_{1}, \delta_{2}, \delta_{3}$ are the eigenvalues $\tilde{\mathcal{K}}^{(\lambda, \boldsymbol{\mu})}(0)$ then we know from [7, Proposition 2.20] that $\delta_{i}>0$ for $i=1,2,3$. Now suppose that $\left(\delta_{1}, \delta_{2}, \delta_{3}\right)$ is a fixed ordered triple of positive numbers. Then there exists a $\mathbf{B}^{(\lambda, \boldsymbol{\mu})}$ with $\lambda>1$ and $\mu_{\ell}>0(\ell=1,2)$ such that $\tilde{\mathcal{K}}^{(\lambda, \mu)}(0)=\operatorname{diag}\left(\delta_{1}, \delta_{2}, \delta_{3}\right)$ only if all $\delta_{i}$ the satisfy the inequalities of Lemma 3.8.

Suppose $\left(\delta_{1}, \delta_{2}, \delta_{3}\right), \delta_{i}>0$, for $i=1,2,3$ is given satisfying the inequalities of Lemma 3.8. Then let us find $\lambda>1, \mu_{1}, \mu_{2}>0$ such that $\tilde{\mathcal{K}}^{(\lambda, \boldsymbol{\mu})}(0)=\operatorname{diag}\left(\delta_{1}, \delta_{2}, \delta_{3}\right)$ with $\boldsymbol{\mu}=\left(1, \mu_{1}, \mu_{2}\right)^{\operatorname{tr}}$. We have $L(\lambda) \boldsymbol{\mu}^{2}=\boldsymbol{d}$, which is the same as

$$
\boldsymbol{\mu}^{2}=L(\lambda)^{-1} \boldsymbol{d}=\left(\begin{array}{ccc}
1 & 0 & 0 \\
-\frac{1}{2(\lambda-1)} & 1 & 0 \\
\frac{1}{\lambda(2 \lambda-1)} & -\frac{2}{\lambda} & 1
\end{array}\right)\left(\begin{array}{c}
1 \\
d_{1} \\
d_{2}
\end{array}\right)=\left(\begin{array}{c}
1 \\
d_{1}-\frac{1}{2(\lambda-1)} \\
2 d_{1}+\frac{1}{\lambda(2 \lambda-1)}
\end{array}\right)
$$

Thus

$$
\mu_{1}^{2}=d_{1}-\frac{1}{2(\lambda-1)}=\frac{1}{\alpha_{1}}-\frac{1}{2(\lambda-1)}=\frac{2(\lambda-1)-\alpha_{1}}{2 \alpha_{1}(\lambda-1)} .
$$

Recall from Lemma 3.8 that

$$
2 \lambda_{0}=2 \lambda-2=\frac{\delta_{1}+\delta_{2}+\delta_{3}}{3}-2 \quad \text { and } \quad \alpha_{1}=\frac{\delta_{2}+\delta_{3}-2 \delta_{1}-6}{3} .
$$

Therefore, we have

$$
2(\lambda-1)-\alpha_{1}=\frac{\delta_{1}+\delta_{2}+\delta_{3}}{3}-2-\frac{\delta_{2}+\delta_{3}-2 \delta_{1}-6}{3}=\delta_{1}>0 .
$$

Similarly

$$
\mu_{2}^{2}=d_{2}-\frac{2 d_{1}}{\lambda}+\frac{1}{\lambda(2 \lambda-1)}=\frac{4}{\alpha_{1} \alpha_{2}}-\frac{2}{\alpha_{1} \lambda}+\frac{1}{\lambda(2 \lambda-1)}=\frac{2\left(2 \lambda-\alpha_{2}\right)(2 \lambda-1)+\alpha_{1} \alpha_{2}}{\alpha_{1} \alpha_{2} \lambda(2 \lambda-1)},
$$

where $\alpha_{1}, \alpha_{2}$ are as in Lemma 3.3. Consequently, we have the following theorem.

Theorem 4.2. There exists a $\mathbf{B}^{(\lambda, \boldsymbol{\mu})}$ such that $\tilde{\mathcal{K}}^{(\lambda, \boldsymbol{\mu})}(0)=\operatorname{diag}\left(\delta_{1}, \delta_{2}, \delta_{3}\right)$ for some $\delta_{1}, \delta_{2}, \delta_{3}>0$ if

$$
\begin{gathered}
\delta_{1}+\delta_{2}+\delta_{3}>6 \\
\delta_{2}+\delta_{3}-2 \delta_{1}>6 \\
2 \delta_{3}-\delta_{1}-\delta_{2}>6 \\
2\left(2 \lambda-\alpha_{2}\right)(2 \lambda-1)+\alpha_{1} \alpha_{2}>0
\end{gathered}
$$

where $\alpha_{1}, \alpha_{2}$ are as in Notation 3.4. 
Notation 4.3. From now on, we will adhere to the following notational convention (here, $(\lambda, \boldsymbol{\mu})$ is fixed but arbitrary).

$$
\begin{aligned}
(\lambda, \boldsymbol{\mu}) & : \mathcal{K}^{(\lambda, \boldsymbol{\mu})}(0)=\operatorname{diag}\left(\delta_{1}, \delta_{2}, \delta_{3}\right), \\
\left(\lambda^{\prime}, \boldsymbol{\mu}^{\prime}\right) & : \mathcal{K}^{\left(\lambda^{\prime}, \boldsymbol{\mu}^{\prime}\right)}(0)=\operatorname{diag}\left(\delta_{2}, \delta_{1}, \delta_{3}\right) \\
(\widehat{\lambda}, \widehat{\boldsymbol{\mu}}) & : \mathcal{K}^{(\widehat{\lambda}, \widehat{\boldsymbol{\mu}})}(0)=\operatorname{diag}\left(\delta_{1}, \delta_{3}, \delta_{2}\right) .
\end{aligned}
$$

Proposition 4.4. Suppose $\delta_{i}>0$ for $i=1,2,3$ are such that $\delta_{1} \neq \delta_{2}$ and $2\left(\delta_{1}+\delta_{2}\right)>\delta_{3}-$ $6>\max \left\{2 \delta_{1}-\delta_{2}, 2 \delta_{2}-\delta_{1}\right\}$. Then there exist reproducing kernels $\mathbf{B}^{(\lambda, \boldsymbol{\mu})}$ and $\mathbf{B}^{\left(\lambda^{\prime}, \boldsymbol{\mu}^{\prime}\right)}$ such that $\tilde{\mathcal{K}}^{(\lambda, \boldsymbol{\mu})}(0)=\operatorname{diag}\left(\delta_{1}, \delta_{2}, \delta_{3}\right)$ and $\tilde{\mathcal{K}}^{\left(\lambda^{\prime}, \boldsymbol{\mu}^{\prime}\right)}(0)=\operatorname{diag}\left(\delta_{2}, \delta_{1}, \delta_{3}\right)$, where $\lambda, \lambda^{\prime}>1, \boldsymbol{\mu}=\left(1, \mu_{1}, \mu_{2}\right)^{\mathrm{tr}}$, $\boldsymbol{\mu}^{\prime}=\left(1, \mu_{1}^{\prime}, \mu_{2}^{\prime}\right)^{\operatorname{tr}}, \mu_{\ell}, \mu_{\ell}^{\prime}>0$ for $\ell=1,2$.

Proof. Consider $\left(\delta_{1}, \delta_{2}, \delta_{3}\right), \delta_{i}>0$ for $i=1,2,3$ such that there exist $\mathbf{B}^{(\lambda, \boldsymbol{\mu})}$ and $\tilde{\mathcal{K}}^{(\lambda, \boldsymbol{\mu})}(0)=$ $\operatorname{diag}\left(\delta_{1}, \delta_{2}, \delta_{3}\right)$ for some $\lambda>1, \boldsymbol{\mu}=\left(1, \mu_{1}, \mu_{2}\right)^{\text {tr }}$ with $\mu_{1}, \mu_{2}>0$. Hence, $\delta_{1}, \delta_{2}, \delta_{3}$ satisfy the inequalities of Lemma 3.8. We now produce $\lambda^{\prime}>1, \boldsymbol{\mu}^{\prime}=\left(1, \mu_{1}^{\prime}, \mu_{2}^{\prime}\right)^{\text {tr }}$ with $\mu_{1}^{\prime}, \mu_{2}^{\prime}>0$ such that $\tilde{\mathcal{K}}^{\left(\lambda^{\prime}, \boldsymbol{\mu}^{\prime}\right)}(0)=\operatorname{diag}\left(\delta_{2}, \delta_{1}, \delta_{3}\right)$. We recall that $\tilde{\mathcal{K}}^{\left(\lambda^{\prime}, \boldsymbol{\mu}^{\prime}\right)}$ is the curvature of the metric $\tilde{\mathbf{B}}^{\left(\lambda^{\prime}, \boldsymbol{\mu}^{\prime}\right)}(z, z)^{\mathrm{tr}}$ and $\tilde{\mathbf{B}}^{\left(\lambda^{\prime}, \boldsymbol{\mu}^{\prime}\right)}$ denotes the normalization of the reproducing kernel $\mathbf{B}^{\left(\lambda^{\prime}, \boldsymbol{\mu}^{\prime}\right)}$. By Lemma 3.3 and Remark 3.5, we need to consider the equations

$$
\begin{gathered}
2 \lambda^{\prime}-\alpha_{1}^{\prime}-2=\delta_{2}, \\
2 \lambda^{\prime}+\alpha_{1}^{\prime}-\alpha_{2}^{\prime}=\delta_{1}, \\
2 \lambda^{\prime}+\alpha_{2}^{\prime}+2=\delta_{3},
\end{gathered}
$$

where $\alpha_{1}^{\prime}=d_{1}^{\prime-1}, \alpha_{2}^{\prime}=4 d_{1}^{\prime} d_{2}^{\prime-1}$. This is the same as $A \boldsymbol{x}^{\prime}=\boldsymbol{b}^{\prime}$, where $A=\left(\begin{array}{ccc}1 & -1 & 0 \\ 1 & 1 & -1 \\ 1 & 0 & 1\end{array}\right), \boldsymbol{x}^{\prime}=$ $\left(\begin{array}{c}2 \lambda^{\prime} \\ \alpha_{1}^{\prime} \\ \alpha_{2}^{\prime}\end{array}\right), \quad \boldsymbol{b}^{\prime}=\left(\begin{array}{c}\delta_{2}+2 \\ \delta_{1} \\ \delta_{3}-2\end{array}\right)$. This system of linear equations has only one solution, namely, $\boldsymbol{x}=\frac{1}{3}\left(\begin{array}{c}\delta_{1}+\delta_{2}+\delta_{3} \\ \delta_{1}+\delta_{3}-2 \delta_{2}-6 \\ 2 \delta_{3}-\delta_{1}-\delta_{2}-6\end{array}\right)$. We observe from Lemma 3.8 that $\lambda=\lambda^{\prime}$ and $\alpha_{2}=\alpha_{2}^{\prime}$ but $\alpha_{1} \neq \alpha_{1}^{\prime}$ if $\delta_{1} \neq \delta_{2}$. From Lemma 3.8 and Theorem 4.2, we know that there exists a $\mathbf{B}^{\left(\lambda^{\prime}, \boldsymbol{\mu}^{\prime}\right)}$ such that $\tilde{\mathcal{K}}^{\left(\lambda^{\prime}, \boldsymbol{\mu}^{\prime}\right)}(0)=\operatorname{diag}\left(\delta_{2}, \delta_{1}, \delta_{3}\right)$ if

$$
\begin{gathered}
\delta_{1}+\delta_{2}+\delta_{3}>6, \\
\delta_{1}+\delta_{3}-2 \delta_{2}>6, \\
2 \delta_{3}-\delta_{1}-\delta_{2}>6 ; \\
2\left(2 \lambda^{\prime}-\alpha_{2}^{\prime}\right)\left(2 \lambda^{\prime}-1\right)+\alpha_{1}^{\prime} \alpha_{2}^{\prime}>0 .
\end{gathered}
$$

Hence there exist $\mathbf{B}^{(\lambda, \boldsymbol{\mu})}$ and $\mathbf{B}^{\left(\lambda^{\prime}, \boldsymbol{\mu}^{\prime}\right)}$ such that $\tilde{\mathcal{K}}^{(\lambda, \boldsymbol{\mu})}(0)=\operatorname{diag}\left(\delta_{1}, \delta_{2}, \delta_{3}\right)$ and $\tilde{\mathcal{K}}^{\left(\lambda^{\prime}, \boldsymbol{\mu}^{\prime}\right)}(0)=$ $\operatorname{diag}\left(\delta_{2}, \delta_{1}, \delta_{3}\right)$ if

$$
\begin{gathered}
\delta_{1}+\delta_{2}+\delta_{3}>6, \\
\delta_{2}+\delta_{3}-2 \delta_{1}>6, \\
\delta_{1}+\delta_{3}-2 \delta_{2}>6, \\
2 \delta_{3}-\delta_{1}-\delta_{2}>6 ; \\
2\left(2 \lambda-\alpha_{2}\right)(2 \lambda-1)+\alpha_{1} \alpha_{2}>0, \\
2\left(2 \lambda^{\prime}-\alpha_{2}^{\prime}\right)\left(2 \lambda^{\prime}-1\right)+\alpha_{1}^{\prime} \alpha_{2}^{\prime}>0 .
\end{gathered}
$$


Suppose that $\delta_{i}>0$ for $i=1,2,3$ are chosen such that $\delta_{1} \neq \delta_{2}$ and

$$
\text { (i) } 2\left(\delta_{1}+\delta_{2}\right)>\delta_{3}-6>\max \left\{2 \delta_{1}-\delta_{2}, 2 \delta_{2}-\delta_{1}\right\} \text {. }
$$

Then the last part of the inequality (i) is clearly seen to force the two inequalities $\delta_{2}+\delta_{3}-$ $2 \delta_{1}>6$ and $\delta_{1}+\delta_{3}-2 \delta_{2}>6$. Adding these two inequalities, we have $2 \delta_{3}-\delta_{1}-\delta_{2}>12$. This choice of $\delta_{i}, i=1,2,3$, also implies $\delta_{3}>6$. Consequently, the first four of the six inequalities listed above are valid. Since $\lambda=\lambda^{\prime}$ and $\alpha_{2}=\alpha_{2}^{\prime}, 2 \lambda^{\prime}-1=2 \lambda-1>0$, it follows from the first part of inequality (i) that $2 \lambda^{\prime}-\alpha_{2}^{\prime}=2 \lambda-\alpha_{2}=\frac{1}{3}\left(2\left(\delta_{1}+\delta_{2}\right)-\delta_{3}\right)+2>0$. Thus the last two inequalities of the six inequalities listed above are valid with our choice of the $\delta_{i}, i=1,2,3$. Hence all the inequalities we need for the simultaneous existence of $\mathbf{B}^{(\lambda, \boldsymbol{\mu})}$ and $\mathbf{B}^{\left(\lambda^{\prime}, \boldsymbol{\mu}^{\prime}\right)}$ are verified by this choice of $\delta_{i}>0$ for $i=1,2,3$.

Proposition 4.5. Suppose that $\delta_{i}>0$ for $i=1,2,3$ are such that $\delta_{3}>\delta_{2}>3+\delta_{3} / 2$ and $\delta_{1}<\min \left\{2 \delta_{3}-\delta_{2}, 2 \delta_{2}-\delta_{3}\right\}-6$. Then there exist reproducing kernels $\mathbf{B}^{(\lambda, \boldsymbol{\mu})}$ and $\mathbf{B}^{(\widehat{\lambda}, \widehat{\boldsymbol{\mu}})}$ such that $\tilde{\mathcal{K}}^{(\lambda, \boldsymbol{\mu})}(0)=\operatorname{diag}\left(\delta_{1}, \delta_{2}, \delta_{3}\right)$ and $\tilde{\mathcal{K}}^{(\widehat{\lambda}, \widehat{\boldsymbol{\mu}})}(0)=\operatorname{diag}\left(\delta_{1}, \delta_{3}, \delta_{2}\right)$, where $\lambda, \widehat{\lambda}>1, \boldsymbol{\mu}=$ $\left(1, \mu_{1}, \mu_{2}\right)^{\operatorname{tr}}, \widehat{\boldsymbol{\mu}}=\left(1, \widehat{\mu}_{1}, \widehat{\mu}_{2}\right)^{\operatorname{tr}}, \mu_{\ell}, \widehat{\mu}_{\ell}>0$ for $\ell=1,2$.

Proof. We construct a reproducing kernel $\mathbf{B}^{(\widehat{\lambda}, \widehat{\boldsymbol{\mu}})}$ such that $\tilde{\mathcal{K}}^{(\widehat{\lambda}, \widehat{\boldsymbol{\mu}})}(0)=\operatorname{diag}\left(\delta_{1}, \delta_{3}, \delta_{2}\right)$ for some $\widehat{\lambda}>1, \widehat{\boldsymbol{\mu}}=\left(1, \widehat{\mu}_{1}, \widehat{\mu}_{2}\right)^{\operatorname{tr}}, \widehat{\mu}_{\ell}>0$ for $\ell=1,2$. By Lemma 3.3 and Remark 3.5, we obtain $\left(2 \widehat{\lambda}, \widehat{\alpha}_{1}, \widehat{\alpha}_{2}\right)$ from the following set of equations:

$$
\begin{gathered}
2 \widehat{\lambda}-\widehat{\alpha}_{1}-2=\delta_{1}, \\
2 \widehat{\lambda}+\widehat{\alpha}_{1}-\widehat{\alpha}_{2}=\delta_{3}, \\
2 \widehat{\lambda}+\widehat{\alpha}_{2}+2=\delta_{2},
\end{gathered}
$$

where $\widehat{\alpha}_{1}=\widehat{d}_{1}^{-1}, \widehat{\alpha}_{2}=4 \widehat{d}_{1} \widehat{d}_{2}^{-1}$. This is same as $A \widehat{\boldsymbol{x}}=\widehat{\boldsymbol{b}}$, where $A=\left(\begin{array}{ccc}1 & -1 & 0 \\ 1 & 1 & -1 \\ 1 & 0 & 1\end{array}\right), \widehat{\boldsymbol{x}}=\left(\begin{array}{c}2 \widehat{\lambda} \\ \widehat{\alpha}_{1} \\ \widehat{\alpha}_{2}\end{array}\right)$, $\widehat{\boldsymbol{b}}=\left(\begin{array}{c}\delta_{2}+2 \\ \delta_{1} \\ \delta_{3}-2\end{array}\right)$. The vector $\boldsymbol{x}=\frac{1}{3}\left(\begin{array}{c}\delta_{1}+\delta_{2}+\delta_{3}, \\ \delta_{2}+\delta_{3}-2 \delta_{1}-6, \\ 2 \delta_{2}-\delta_{1}-\delta_{3}-6\end{array}\right)$ is the only solution of this system of equations. From Lemma 3.3 and Theorem 3.5, we know that there exists a $\mathbf{B}^{(\widehat{\lambda}, \widehat{\boldsymbol{\mu}})}$ such that $\tilde{\mathcal{K}}^{(\widehat{\lambda}, \widehat{\boldsymbol{\mu}})}(0)=$ $\operatorname{diag}\left(\delta_{1}, \delta_{3}, \delta_{2}\right)$ if

$$
\begin{gathered}
\delta_{1}+\delta_{2}+\delta_{3}>6, \\
\delta_{2}+\delta_{3}-2 \delta_{1}>6, \\
2 \delta_{2}-\delta_{1}-\delta_{3}>6 ; \\
2\left(2 \widehat{\lambda}-\widehat{\alpha}_{2}\right)(2 \widehat{\lambda}-1)+\widehat{\alpha}_{1} \widehat{\alpha}_{2}>0
\end{gathered}
$$

If $\left(\delta_{1}, \delta_{2}, \delta_{3}\right), \delta_{i}>0$ for $i=1,2,3$ are such that there exist $\mathbf{B}^{(\lambda, \boldsymbol{\mu})}$ and $\tilde{\mathcal{K}}^{(\lambda, \boldsymbol{\mu})}(0)=$ $\operatorname{diag}\left(\delta_{1}, \delta_{2}, \delta_{3}\right)$, then the $\delta_{i}$ for $i=1,2,3$ satisfies the inequalities of Lemma 3.8. Hence there exist $\mathbf{B}^{(\lambda, \boldsymbol{\mu})}$ and $\mathbf{B}^{(\widehat{\lambda}, \widehat{\boldsymbol{\mu}})}$ simultaneously such that $\tilde{\mathcal{K}}^{(\lambda, \boldsymbol{\mu})}(0)=\operatorname{diag}\left(\delta_{1}, \delta_{2}, \delta_{3}\right)$ and $\tilde{\mathcal{K}}^{(\widehat{\lambda}, \widehat{\boldsymbol{\mu}})}(0)=$ $\operatorname{diag}\left(\delta_{1}, \delta_{3}, \delta_{2}\right)$ if

$$
\begin{gathered}
\delta_{1}+\delta_{2}+\delta_{3}>6, \\
\delta_{2}+\delta_{3}-2 \delta_{1}>6, \\
2 \delta_{2}-\delta_{1}-\delta_{3}>6,
\end{gathered}
$$




$$
\begin{gathered}
2 \delta_{3}-\delta_{1}-\delta_{2}>6 \\
2\left(2 \lambda-\alpha_{2}\right)(2 \lambda-1)+\alpha_{1} \alpha_{2}>0 \\
2\left(2 \widehat{\lambda}-\widehat{\alpha}_{2}\right)(2 \widehat{\lambda}-1)+\widehat{\alpha}_{1} \widehat{\alpha}_{2}>0 .
\end{gathered}
$$

We observe that $\lambda=\widehat{\lambda}$ and $\alpha_{1}=\widehat{\alpha}_{1}$ but $\alpha_{2} \neq \widehat{\alpha}_{2}$ if $\delta_{2} \neq \delta_{3}$. Suppose that $\delta_{i}>0$ for $i=1,2,3$ are chosen satisfying

$$
\text { (a) } \delta_{3}>\delta_{2}>3+\frac{\delta_{3}}{2} \text { and (b) } \delta_{1}<\min \left\{2 \delta_{3}-\delta_{2}, 2 \delta_{2}-\delta_{3}\right\}-6 \text {. }
$$

Then inequality (a) implies that $\delta_{3}>6$, and hence the first of the set of six inequalities above holds. Inequality (b) implies that $2 \delta_{3}-\delta_{1}-\delta_{2}>6$; and $2 \delta_{2}-\delta_{1}-\delta_{3}>6$; adding these two inequalities we have $\delta_{2}+\delta_{3}-2 \delta_{1}>12$. Hence the first four inequalities, from the list of six inequalities given above, are verified. The second, third and the second, fourth from the set of the six inequalities, respectively, imply that $\delta_{2}-\delta_{1}>4$ and $\delta_{3}-\delta_{1}>4$. An easy computation involving the expressions for $\lambda, \alpha_{1}, \alpha_{2}$ and $\hat{\lambda}, \widehat{\alpha}_{1}, \widehat{\alpha}_{2}$ in terms of $\delta_{i}$ for $i=1,2,3$ shows that $2\left(2 \lambda-\alpha_{2}\right)(2 \lambda-1)+\alpha_{1} \alpha_{2}>0$ and $2\left(2 \widehat{\lambda}-\widehat{\alpha}_{2}\right)(2 \widehat{\lambda}-1)+\widehat{\alpha}_{1} \widehat{\alpha}_{2}>0$ together is equivalent to $\left(\delta_{1}+\delta_{2}\right)\left(2 \delta_{1}+\delta_{2}\right)+\delta_{3}\left(\delta_{2}-\delta_{1}\right)+6 \delta_{1}>0$ and $\left(\delta_{1}+\delta_{3}\right)\left(2 \delta_{1}+\delta_{3}\right)+$ $\delta_{2}\left(\delta_{3}-\delta_{1}\right)+6 \delta_{1}>0$. These are satisfied as $\delta_{2}-\delta_{1}>4$ and $\delta_{3}-\delta_{1}>4$. Hence all the required inequalities for the simultaneous existence of $\mathbf{B}^{(\lambda, \mu)}$ and $\mathbf{B}^{(\widehat{\lambda}, \widehat{\boldsymbol{\mu}})}$ are met by this choice of $\delta_{i}>0$ for $i=1,2,3$.

REMARK 4.6. The set $\left\{\delta_{i}>0: i=1,2,3\right\}$ satisfying the inequalities of Proposition 4.4 is non-empty. For instance, take $\delta_{1}=1, \delta_{2}=2$ and any $\delta_{3}$ in the open interval $(9,12)$. Then $\left\{\delta_{1}, \delta_{2}, \delta_{3}\right\}$ meets the requirement. Similarly, taking any $\delta_{1}$ in the open interval $(0,1), \delta_{2}=7.5$ and $\delta_{3}=8$, we find that $\left\{\delta_{1}, \delta_{2}, \delta_{3}\right\}$ satisfies the inequalities prescribed in Proposition 4.5. Thus the two sets which are obtained from Propositions 4.4 and 4.5 are not identical.

Corollary 4.7. In Propositions 4.4 and $4.5,\left(\begin{array}{l}\lambda \\ \boldsymbol{\mu}\end{array}\right) \neq\left(\begin{array}{c}\lambda^{\prime} \\ \boldsymbol{\mu}^{\prime}\end{array}\right)$ and $\left(\begin{array}{c}\lambda \\ \boldsymbol{\mu}\end{array}\right) \neq\left(\begin{array}{c}\widehat{\lambda} \\ \hat{\boldsymbol{\mu}}\end{array}\right)$.

Proof. By Lemma 3.9, it suffices to show that $\left(2 \lambda, \alpha_{1}, \alpha_{2}\right) \neq\left(2 \lambda^{\prime}, \alpha_{1}^{\prime}, \alpha_{2}^{\prime}\right)$ and $\left(2 \lambda, \alpha_{1}, \alpha_{2}\right) \neq$ $\left(2 \widehat{\lambda}, \widehat{\alpha}_{1}, \widehat{\alpha}_{2}\right)$. However, in Proposition $4.4, \alpha_{1} \neq \alpha_{1}^{\prime}$ since $\delta_{1} \neq \delta_{2}$. Similarly, in Proposition 4.5, $\alpha_{2} \neq \widehat{\alpha}_{2}$ since $\delta_{2} \neq \delta_{3}$.

Recall that $M^{(\lambda, \boldsymbol{\mu})}$ denotes the multiplication operator on the reproducing kernel Hilbert spaces with reproducing kernel $\mathbf{B}^{(\lambda, \boldsymbol{\mu})}$.

Corollary 4.8. Suppose that $\mathbf{B}^{(\lambda, \boldsymbol{\mu})}, \mathbf{B}^{\left(\lambda^{\prime}, \boldsymbol{\mu}^{\prime}\right)}$ and $\mathbf{B}^{(\lambda, \boldsymbol{\mu})}, \mathbf{B}^{(\widehat{\lambda}, \widehat{\boldsymbol{\mu}})}$ are as in Propositions 4.4 and 4.5, respectively. Then we have:

(a) the multiplication operators $M^{(\lambda, \boldsymbol{\mu})}$ and $M^{\left(\lambda^{\prime}, \boldsymbol{\mu}^{\prime}\right)}$ are not unitarily equivalent;

(b) the multiplication operators $M^{(\lambda, \mu)}$ and $M^{(\widehat{\lambda}, \widehat{\mu})}$ are not unitarily equivalent.

Proof. The proof is immediate from Theorem 3.11 and Corollary 4.7.

Remark 4.9. In Propositions 4.4 and 4.5, we have shown the following: Given a reproducing kernel $\mathbf{B}^{(\lambda, \boldsymbol{\mu})}$ such that $\tilde{\mathcal{K}}^{(\lambda, \boldsymbol{\mu})}(0)=\operatorname{diag}\left(\delta_{1}, \delta_{2}, \delta_{3}\right)$ there exists a reproducing kernel $\mathbf{B}^{\left(\lambda^{\prime}, \boldsymbol{\mu}^{\prime}\right)}$ with $\left(\begin{array}{c}\lambda \\ \boldsymbol{\mu}\end{array}\right) \neq\left(\begin{array}{c}\lambda^{\prime} \\ \boldsymbol{\mu}^{\prime}\end{array}\right)$ such that $\tilde{\mathcal{K}}^{\left(\lambda^{\prime}, \boldsymbol{\mu}^{\prime}\right)}(0)=\operatorname{diag}\left(\delta_{\rho(1)}, \delta_{\rho(2)}, \delta_{\rho(3)}\right)$, and given a reproducing kernel $\mathbf{B}^{(\lambda, \boldsymbol{\mu})}$ such that $\tilde{\mathcal{K}}^{(\lambda, \boldsymbol{\mu})}(0)=\operatorname{diag}\left(\delta_{1}, \delta_{2}, \delta_{3}\right)$ there exists a reproducing 
kernel $\mathbf{B}^{(\widehat{\lambda}, \widehat{\boldsymbol{\mu}})}$ with $\left(\begin{array}{c}\lambda \\ \boldsymbol{\mu}\end{array}\right) \neq\left(\begin{array}{c}\widehat{\lambda} \\ \widehat{\boldsymbol{\mu}}\end{array}\right)$ such that $\tilde{\mathcal{K}}^{(\widehat{\lambda}, \widehat{\boldsymbol{\mu}})}(0)=\operatorname{diag}\left(\delta_{\tau(1)}, \delta_{\tau(2)}, \delta_{\tau(3)}\right)$, where $\rho, \tau \in S_{3}$ with $\rho(1)=2, \rho(2)=1, \rho(3)=3, \tau(1)=1, \tau(2)=3, \tau(3)=2$. In the next proposition we prove that if there exists a reproducing kernel $\mathbf{B}^{(\lambda, \boldsymbol{\mu})}$ such that $\tilde{\mathcal{K}}^{(\lambda, \boldsymbol{\mu})}(0)=\operatorname{diag}\left(\delta_{1}, \delta_{2}, \delta_{3}\right)$ there does not exist $\mathbf{B}^{(\vartheta, \boldsymbol{\xi})}$ with $\left(\begin{array}{c}\lambda \\ \boldsymbol{\mu}\end{array}\right) \neq\left(\begin{array}{c}\vartheta \\ \boldsymbol{\xi}\end{array}\right)$ such that $\tilde{\mathcal{K}}^{(\vartheta, \boldsymbol{\xi})}(0)=\operatorname{diag}\left(\delta_{\sigma(1)}, \delta_{\sigma(2)}, \delta_{\sigma(3)}\right)$ unless $\sigma=\rho$ or $\tau$, for $\sigma, \rho, \tau \in S_{3}$. Obviously, there exists $\mathbf{B}_{\sigma}^{(\lambda, \boldsymbol{\mu})}:=P_{\sigma} \mathbf{B}^{(\lambda, \boldsymbol{\mu})} P_{\sigma}^{*}$ such that $\tilde{\mathcal{K}}_{\sigma}(0)=\operatorname{diag}$ $\left(\delta_{\sigma(1)}, \delta_{\sigma(2)}, \delta_{\sigma(3)}\right)$ for all $\sigma \in S_{3}$, where $P_{\sigma}$ is in $\mathcal{M}_{3}$ such that $P_{\sigma}(i, j)=\delta_{\sigma(i), j}$ and $\tilde{\mathcal{K}}_{\sigma}$ is the curvature with respect to the metric $\tilde{h}_{\sigma}(z)=\tilde{\mathbf{B}}_{\sigma}^{(\lambda, \boldsymbol{\mu})}(z, z)^{\mathrm{tr}}$. The reproducing kernels $\mathbf{B}^{(\lambda, \boldsymbol{\mu})}$ and $\mathbf{B}_{\sigma}^{(\lambda, \boldsymbol{\mu})}$ are equivalent; that is, the multiplication operators on the reproducing kernel Hilbert spaces with reproducing kernels $\mathbf{B}^{(\lambda, \boldsymbol{\mu})}$ and $\mathbf{B}_{\sigma}^{(\lambda, \boldsymbol{\mu})}$ are unitarily equivalent. Therefore, we do not distinguish between the two reproducing kernels $\mathbf{B}^{(\lambda, \boldsymbol{\mu})}$ and $\mathbf{B}_{\sigma}^{(\lambda, \boldsymbol{\mu})}$.

Proposition 4.10. Given a reproducing kernel $\mathbf{B}^{(\lambda, \boldsymbol{\mu})}$ such that $\tilde{\mathcal{K}}^{(\lambda, \boldsymbol{\mu})}(0)=$ $\operatorname{diag}\left(\delta_{1}, \delta_{2}, \delta_{3}\right)$, there does not exist a reproducing kernel $\mathbf{B}^{(\vartheta, \boldsymbol{\xi})}$ such that $\tilde{\mathcal{K}}^{(\vartheta, \boldsymbol{\xi})}(0)=$ $\operatorname{diag}\left(\delta_{\sigma(1)}, \delta_{\sigma(2)}, \delta_{\sigma(3)}\right)$ with $\left(\begin{array}{c}\lambda \\ \mu\end{array}\right) \neq\left(\begin{array}{l}\vartheta \\ \xi\end{array}\right)$ unless $\sigma=\rho$ or $\sigma=\tau$.

Proof. Case 1: Choose $\sigma \in S_{3}$ such that $\sigma(1)=3, \sigma(2)=2, \sigma(3)=1$.

The existence of two reproducing kernels $\mathbf{B}^{(\lambda, \boldsymbol{\mu})}$ and $\mathbf{B}^{(\vartheta, \boldsymbol{\xi})}$ such that $\tilde{\mathcal{K}}^{(\lambda, \boldsymbol{\mu})}(0)=$ $\operatorname{diag}\left(\delta_{1}, \delta_{2}, \delta_{3}\right)$ and $\tilde{\mathcal{K}}^{(\vartheta, \boldsymbol{\xi})}(0)=\operatorname{diag}\left(\delta_{\sigma(1)}, \delta_{\sigma(2)}, \delta_{\sigma(3)}\right)$ would imply, by an application of Lemma 3.8 to the ordered triples $\left(\delta_{1}, \delta_{2}, \delta_{3}\right)$ and $\left(\delta_{\sigma(1)}, \delta_{\sigma(2)}, \delta_{\sigma(3)}\right)=\left(\delta_{3}, \delta_{2}, \delta_{1}\right)$ that

$$
\begin{gathered}
\delta_{1}+\delta_{2}+\delta_{3}>6, \\
\delta_{2}+\delta_{3}-2 \delta_{1}>6, \\
2 \delta_{3}-\delta_{1}-\delta_{2}>6 ; \\
\delta_{\sigma(1)}+\delta_{\sigma(2)}+\delta_{\sigma(3)}>6, \\
\delta_{\sigma(2)}+\delta_{\sigma(3)}-2 \delta_{\sigma(1)}>6, \\
2 \delta_{\sigma(3)}-\delta_{\sigma(1)}-\delta_{\sigma(2)}>6 .
\end{gathered}
$$

This set of inequalities are equivalent to

$$
\begin{gathered}
\delta_{1}+\delta_{2}+\delta_{3}>6, \\
\delta_{2}+\delta_{3}-2 \delta_{1}>6, \\
2 \delta_{3}-\delta_{1}-\delta_{2}>6, \\
\delta_{1}+\delta_{2}-2 \delta_{3}>6, \\
2 \delta_{1}-\delta_{2}-\delta_{3}>6 .
\end{gathered}
$$

Adding the third and the fourth from these inequalities gives $0>12$.

Case 2: Choose $\sigma \in S_{3}$ such that $\sigma(1)=2, \sigma(2)=3, \sigma(3)=1$.

As in the first case the existence of two reproducing kernels $\mathbf{B}^{(\lambda, \boldsymbol{\mu})}$ and $\mathbf{B}^{(\vartheta, \boldsymbol{\xi})}$ such that $\tilde{\mathcal{K}}^{(\lambda, \boldsymbol{\mu})}(0)=\operatorname{diag}\left(\delta_{1}, \delta_{2}, \delta_{3}\right)$ and $\tilde{\mathcal{K}}^{(\vartheta, \boldsymbol{\xi})}(0)=\operatorname{diag}\left(\delta_{\sigma(1)}, \delta_{\sigma(2)}, \delta_{\sigma(3)}\right)$ would imply, by an application of Lemma 3.8 to the ordered triples $\left(\delta_{1}, \delta_{2}, \delta_{3}\right)$ and $\left(\delta_{\sigma(1)}, \delta_{\sigma(2)}, \delta_{\sigma(3)}\right)=\left(\delta_{2}, \delta_{3}, \delta_{1}\right)$, that

$$
\begin{gathered}
\delta_{1}+\delta_{2}+\delta_{3}>6, \\
\delta_{2}+\delta_{3}-2 \delta_{1}>6, \\
2 \delta_{3}-\delta_{1}-\delta_{2}>6, \\
\delta_{1}+\delta_{3}-2 \delta_{2}>6, \\
2 \delta_{1}-\delta_{2}-\delta_{3}>6 .
\end{gathered}
$$

Adding second and fifth of these inequalities gives $0>12$. 
Case 3: Take $\sigma \in S_{3}$ such that $\sigma(1)=3, \sigma(2)=1, \sigma(3)=2$.

Finally, continuing in the same manner in the previous two cases, the existence of two reproducing kernels $\mathbf{B}^{(\lambda, \boldsymbol{\mu})}$ and $\mathbf{B}^{(\vartheta, \boldsymbol{\xi})}$ such that $\tilde{\mathcal{K}}^{(\lambda, \boldsymbol{\mu})}(0)=\operatorname{diag}\left(\delta_{1}, \delta_{2}, \delta_{3}\right)$ and $\tilde{\mathcal{K}}^{(\vartheta, \boldsymbol{\xi})}(0)=$ $\operatorname{diag}\left(\delta_{\sigma(1)}, \delta_{\sigma(2)}, \delta_{\sigma(3)}\right)$ would imply, by an application of Lemma 3.8 to the ordered triples $\left(\delta_{1}, \delta_{2}, \delta_{3}\right)$ and $\left(\delta_{\sigma(1)}, \delta_{\sigma(2)}, \delta_{\sigma(3)}\right)=\left(\delta_{3}, \delta_{1}, \delta_{2}\right)$, that

$$
\begin{gathered}
\delta_{1}+\delta_{2}+\delta_{3}>6, \\
\delta_{2}+\delta_{3}-2 \delta_{1}>6, \\
2 \delta_{3}-\delta_{1}-\delta_{2}>6, \\
\delta_{1}+\delta_{2}-2 \delta_{3}>6, \\
2 \delta_{2}-\delta_{3}-\delta_{1}>6 .
\end{gathered}
$$

Adding third and fourth inequalities from this set of inequalities, we have $0>12$.

Corollary 4.11. There does not exist any multiplication operator $M^{(\vartheta, \boldsymbol{\xi})}$ other than $M^{\left(\lambda^{\prime}, \boldsymbol{\mu}^{\prime}\right)}$ or $M^{(\widehat{\lambda}, \widehat{\boldsymbol{\mu}})}$ such that the sets of eigenvalues of $\tilde{\mathcal{K}}^{(\vartheta, \boldsymbol{\xi})}(0)$ and $\tilde{\mathcal{K}}^{(\lambda, \boldsymbol{\mu})}(0)$ are equal but $\tilde{\mathcal{K}}^{(\vartheta, \boldsymbol{\xi})}(0) \neq \tilde{\mathcal{K}}^{(\lambda, \boldsymbol{\mu})}(0)$, where $\mathbf{B}^{(\lambda, \boldsymbol{\mu})}, \mathbf{B}^{(\tilde{\lambda}, \tilde{\boldsymbol{\mu}})}, \mathbf{B}^{(\widehat{\lambda}, \widehat{\boldsymbol{\mu}})}$ are as in Propositions 4.4 and 4.5.

Proof. Combining Corollary 4.8, Theorem 3.11, Corollary 3.10 and Proposition 4.10, we obtain a proof of this corollary.

REMARK 4.12. We discuss the case $m=1$. From Lemma 3.3, we see that $\tilde{\mathcal{K}}^{(\lambda, \boldsymbol{\mu})}(0)=$ $\operatorname{diag}\left(2 \lambda-\alpha_{1}-1,2 \lambda+\alpha_{1}+1\right)$, where $\lambda>1 / 2, \boldsymbol{\mu}=\left(1, \mu_{1}\right), \mu_{1}>0, \alpha_{1}=d_{1}{ }^{-1}, d_{1}$ is defined as before. If $\tilde{\mathcal{K}}^{(\lambda, \boldsymbol{\mu})}(0)=\operatorname{diag}\left(\delta_{1}, \delta_{2}\right), \delta_{i}>0$ for $i=1,2$, for some $\lambda>1 / 2$ and $\boldsymbol{\mu}=\left(1, \mu_{1}\right), \mu_{1}>0$. Then arguing as in Lemma 3.3, one notes that $2 \lambda=\left(\delta_{1}+\delta_{2}\right) / 2, \alpha_{1}=\left(\delta_{2}-\delta_{1}-2\right) / 2$. As $2 \lambda>1$ and $\alpha_{1}=d^{-1}>0$ it follows that $\delta_{1}+\delta_{2}>2$ and $\delta_{2}-\delta_{1}>2$ are necessary conditions for existence of a reproducing kernel $\mathbf{B}^{(\lambda, \boldsymbol{\mu})}$ such that $\tilde{\mathcal{K}}^{(\lambda, \boldsymbol{\mu})}(0)=\operatorname{diag}\left(\delta_{1}, \delta_{2}\right)$. If $\delta_{i}>0$ for $i=1,2$, proceeding as in Theorem 4.2, one observes that $\delta_{2}-\delta_{1}>2, \delta_{1}+\delta_{2}>2$ and $d_{1}>1 /(2 \lambda-1)=$ $2 /\left(\delta_{1}+\delta_{2}-2\right)$ are the sufficient conditions for existence of a reproducing kernel $\mathbf{B}^{(\lambda, \boldsymbol{\mu})}$ such that $\tilde{\mathcal{K}}^{(\lambda, \boldsymbol{\mu})}(0)=\operatorname{diag}\left(\delta_{1}, \delta_{2}\right)$. Conversely, if $\delta_{i}>0$ for $i=1,2$ and $\delta_{2}-\delta_{1}>2$ then clearly $\delta_{1}+$ $\delta_{2}>2$ and $d_{1}=2 /\left(\delta_{2}-\delta_{1}-2\right)>2 /\left(\delta_{1}+\delta_{2}-2\right)$. Therefore, $\delta_{i}>0$ for $i=1,2$ and $\delta_{2}-\delta_{1}>2$ are the necessary and sufficient conditions for the existence of reproducing kernel $\mathbf{B}^{(\lambda, \boldsymbol{\mu})}$ such that $\tilde{\mathcal{K}}^{(\lambda, \boldsymbol{\mu})}(0)=\operatorname{diag}\left(\delta_{1}, \delta_{2}\right)$.

REMARK 4.13. If $\delta_{i}>0$ for $i=1,2$ and $\delta_{2}-\delta_{1}>2$, there does not exist a reproducing kernel $\mathbf{B}^{(\vartheta, \boldsymbol{\xi})}$ other than $\mathbf{B}^{(\lambda, \boldsymbol{\mu})}$ (up to equivalence as discussed in Remark 4.9) such that $\tilde{\mathcal{K}}^{(\vartheta, \boldsymbol{\xi})}(0)=\operatorname{diag}\left(\delta_{2}, \delta_{1}\right)$. If $\mathbf{B}^{(\vartheta, \boldsymbol{\xi})}$ exists satisfying the above requirements, then from Remark 4.12 we see that both of $\delta_{2}-\delta_{1}>2$ and $\delta_{1}-\delta_{2}>2$ have to be simultaneously satisfied. This is impossible. Hence there does not exist inequivalent multiplication operators $M^{(\lambda, \mu)}$ and $M^{(\vartheta, \boldsymbol{\xi})}$ such that the set of eigenvalues of $\tilde{\mathcal{K}}^{(\vartheta, \boldsymbol{\xi})}(0)$ equals those of $\tilde{\mathcal{K}}^{(\lambda, \boldsymbol{\mu})}(0)$ but $\tilde{\mathcal{K}}^{(\vartheta, \boldsymbol{\xi})}(0) \neq \tilde{\mathcal{K}}^{(\lambda, \boldsymbol{\mu})}(0)$.

Theorem 4.14. Suppose that $\mathbf{B}^{(\lambda, \boldsymbol{\mu})}, \mathbf{B}^{\left(\lambda^{\prime}, \boldsymbol{\mu}^{\prime}\right)}$ and $\mathbf{B}^{(\lambda, \boldsymbol{\mu})}, \mathbf{B}^{(\widehat{\lambda}, \widehat{\boldsymbol{\mu}})}$ are as in Propositions 4.4 and 4.5 respectively. Then we have the following:

(i) the multiplication operators $M^{(\lambda, \boldsymbol{\mu})}$ and $M^{\left(\lambda^{\prime}, \boldsymbol{\mu}^{\prime}\right)}$ are not equivalent although $\tilde{\mathcal{K}}^{(\lambda, \boldsymbol{\mu})}(z)$ and $\tilde{\mathcal{K}}^{\left(\lambda^{\prime}, \boldsymbol{\mu}^{\prime}\right)}(z)$ are unitarily equivalent for $z$ in $\mathbb{D}$; 
(ii) the multiplication operators $M^{(\lambda, \boldsymbol{\mu})}$ and $M^{(\widehat{\lambda}, \widehat{\boldsymbol{\mu}})}$ are not equivalent although $\tilde{\mathcal{K}}^{(\lambda, \boldsymbol{\mu})}(z)$ and $\tilde{\mathcal{K}}^{(\widehat{\lambda}, \widehat{\boldsymbol{\mu}})}(z)$ are unitarily equivalent for $z$ in $\mathbb{D}$.

Proof. From Proposition 4.4, we see that the curvatures of the associated bundles have the same set of eigenvalues at zero, namely $\left\{\delta_{1}, \delta_{2}, \delta_{3}\right\}$. Since curvature is self-adjoint, the set of eigenvalues is the complete set of unitary invariants for the curvature. Thus $\tilde{\mathcal{K}}^{(\lambda, \boldsymbol{\mu})}(0)$ and $\tilde{\mathcal{K}}^{\left(\lambda^{\prime}, \boldsymbol{\mu}^{\prime}\right)}(0)$ are unitarily equivalent. Since the operators $M^{(\lambda, \boldsymbol{\mu})}$ and $M^{\left(\lambda^{\prime}, \boldsymbol{\mu}^{\prime}\right)}$ are homogeneous, by an application of Theorem 2.6, we see that $\tilde{\mathcal{K}}^{(\lambda, \boldsymbol{\mu})}(z)$ and $\tilde{\mathcal{K}}^{\left(\lambda^{\prime}, \boldsymbol{\mu}^{\prime}\right)}(z)$ are unitarily equivalent for $z \in \mathbb{D}$. Now (i) follows from part (a) of Corollary 4.8. The proof of part (ii) of this theorem is similar.

The proof of the next theorem will be complete after proving a sequence of lemmas.

Theorem 4.15. Suppose that $M^{(\lambda, \boldsymbol{\mu})}$ and $M^{(\vartheta, \boldsymbol{\xi})}$ are not unitarily equivalent and the two curvatures $\tilde{\mathcal{K}}^{(\lambda, \boldsymbol{\mu})}(z)$ and $\tilde{\mathcal{K}}^{(\vartheta, \boldsymbol{\xi})}(z)$ are unitarily equivalent for $z \in \mathbb{D}$. Then there does not exist any invertible matrix $L$ in $\mathcal{M}_{3}$ satisfying $L \tilde{\mathcal{K}}^{(\lambda, \boldsymbol{\mu})}(0)=\tilde{\mathcal{K}}^{(\vartheta, \boldsymbol{\xi})}(0) L$ for which $L\left(\tilde{\mathcal{K}}^{(\lambda, \boldsymbol{\mu})}\right)_{\bar{z}}(0)=\left(\tilde{\mathcal{K}}^{(\vartheta, \boldsymbol{\xi})}\right)_{\bar{z}}(0) L$ too. In other words, the covariant derivative of order $(0,1)$ detects the inequivalence.

Lemma 4.16. Suppose that there exist reproducing kernels $\mathbf{B}^{(\lambda, \boldsymbol{\mu})}, \mathbf{B}^{\left(\lambda^{\prime}, \boldsymbol{\mu}^{\prime}\right)}$ with $\tilde{\mathcal{K}}^{(\lambda, \boldsymbol{\mu})}(0)=\operatorname{diag}\left(\delta_{1}, \delta_{2}, \delta_{3}\right), \tilde{\mathcal{K}}^{\left(\lambda^{\prime}, \boldsymbol{\mu}^{\prime}\right)}(0)=\operatorname{diag}\left(\delta_{\rho(1)}, \delta_{\rho(2)}, \delta_{\rho(3)}\right), \delta_{1} \neq \delta_{2}$ and $C$ in $\mathcal{M}_{3}$ is such that $C \tilde{\mathcal{K}}^{(\lambda, \mu)}(0)=\tilde{\mathcal{K}}^{\left(\lambda^{\prime}, \mu^{\prime}\right)}(0) C$. Then $C=\left(\left(C(i, j) \delta_{\rho(i), j}\right)\right)$ for $C(i, j) \in \mathbb{C}, i, j=1,2,3$, where $\rho \in S_{3}$ is given by $\rho(1)=2, \rho(2)=1, \rho(3)=3$.

Proof. The proof of this lemma is immediate from Lemma 3.14, once we ensure that $\delta_{1}, \delta_{2}, \delta_{3}$ are distinct. Recalling notation from Lemma 3.3, we write $\delta_{1}=2 \lambda-\alpha_{1}-2, \delta_{2}=2 \lambda+\alpha_{1}-\alpha_{2}$, $\delta_{3}=2 \lambda+\alpha_{2}+2$. Clearly $\delta_{3}-\delta_{1}=\alpha_{1}+\alpha_{2}+4>0$. Recalling notation from Proposition 4.4 , one has $\delta_{2}=2 \lambda^{\prime}-\alpha_{1}^{\prime}-2, \delta_{1}=2 \lambda^{\prime}+\alpha_{1}^{\prime}-\alpha_{2}^{\prime}, \delta_{3}=2 \lambda^{\prime}+\alpha_{2}^{\prime}+2$. Therefore $\delta_{3}-\delta_{2}=$ $\alpha_{1}+\alpha_{2}+4>0$. We have $\delta_{3}>\delta_{1}, \delta_{3}>\delta_{2}$ and $\delta_{1} \neq \delta_{2}$ by hypothesis. Hence the proof is complete.

The proof of the next lemma is similar and is therefore omitted.

Lemma 4.17. Suppose that there exist reproducing kernels $\mathbf{B}^{(\lambda, \boldsymbol{\mu})}, \mathbf{B}^{(\widehat{\lambda}, \widehat{\boldsymbol{\mu}})}$ with $\tilde{\mathcal{K}}^{(\lambda, \boldsymbol{\mu})}(0)=$ $\operatorname{diag}\left(\delta_{1}, \delta_{2}, \delta_{3}\right), \tilde{\mathcal{K}}^{(\widehat{\lambda}, \widehat{\boldsymbol{\mu}})}(0)=\operatorname{diag}\left(\delta_{\tau(1)}, \delta_{\tau(2)}, \delta_{\tau(3)}\right), \delta_{2} \neq \delta_{3}$ and that $C$ in $\mathcal{M}_{3}$ is such that $C \tilde{\mathcal{K}}^{(\lambda, \boldsymbol{\mu})}(0)=\tilde{\mathcal{K}}^{(\widehat{\lambda}, \widehat{\boldsymbol{\mu}})}(0) C$. Then $C=\left(\left(C(i, j) \delta_{\tau(i), j}\right)\right)_{i, j=1}^{3}$ for $C(i, j) \in \mathbb{C}, i, j=1,2,3$, where $\tau \in S_{3}$ is given by $\tau(1)=1, \tau(2)=3, \tau(3)=2$.

Lemma 4.18. Suppose that $C=\left(\left(C(i, j) \delta_{\sigma(i), j}\right)\right)_{i, j=1}^{3}$ for $\sigma=\rho$ or $\tau$ in $S_{3}$. Then $C$ is invertible if and only if $C(i, \sigma(i)) \neq 0$ for $i=1,2,3$ and $\sigma=\rho$ or $\tau$ in $S_{3}$.

Proof. One observes that $\operatorname{det} C \neq 0$ if and only if $C(i, \sigma(i)) \neq 0$ for $i=1,2,3$ and $\sigma=\rho$ or $\tau$ in $S_{3}$. The proof is therefore complete.

The proof of the following lemma is straightforward. We recall that $S\left(c_{1}, \ldots, c_{m}\right)(\ell, p)=$ $c_{\ell} \delta_{p+1, \ell}, 0 \leqslant p, \ell \leqslant m$. 
Lemma 4.19. Suppose that $C=\left(\left(C(i, j) \delta_{\sigma(i), j}\right)\right)_{i, j=1}^{3}, C(i, \sigma(i)) \neq 0$ for $i=1,2,3$ and $\sigma=$ $\rho, \tau$ in $S_{3}$ is such that $C S\left(c_{1}, c_{2}\right)^{\operatorname{tr}}=S\left(\tilde{c}_{1}, \tilde{c}_{2}\right)^{\operatorname{tr} C}$ for $c_{i}, \tilde{c}_{i}$ in $\mathbb{C}, i=1,2$. Then $c_{i}=\tilde{c}_{i}=0$ for $i=1,2$.

LEMmA 4.20. We have that $\left(\tilde{\mathcal{K}}^{(\lambda, \boldsymbol{\mu})}\right)_{\bar{z}}(0)$ is not the zero matrix.

Proof. If possible let $\left(\tilde{\mathcal{K}}^{(\lambda, \boldsymbol{\mu})}\right)_{\bar{z}}(0)=0$. Then it follows from Lemma 3.3 that $-\sqrt{\alpha}_{1}\left(1+\alpha_{1}-\right.$ $\left.\alpha_{2} / 2\right)=-\sqrt{\alpha}_{2}\left(1+\alpha_{2}-\alpha_{1} / 2\right)=0$. Equivalently, $1+\alpha_{1}-\alpha_{2} / 2=1+\alpha_{2}-\alpha_{1} / 2$, as $\alpha_{1}$ and $\alpha_{2}$ are positive. This implies that $\alpha_{1}=\alpha_{2}$. Therefore $\left(\tilde{\mathcal{K}}^{(\lambda, \boldsymbol{\mu})}\right) \bar{z}(0)=0$ implies by an application of Lemma 3.3 that $-\sqrt{\alpha}_{1}\left(1+\alpha_{1} / 2\right)=0$, which is impossible as $\alpha_{1}$ is positive.

Proof of Theorem 4.15. We observe by applying Propositions 4.4, 4.5 and 4.10 that if $M^{(\vartheta, \boldsymbol{\xi})}$ is a multiplication operator not unitarily equivalent to $M^{(\lambda, \boldsymbol{\mu})}$, then $(\vartheta, \boldsymbol{\xi})=\left(\lambda^{\prime}, \boldsymbol{\mu}^{\prime}\right)$ or $(\widehat{\lambda}, \widehat{\boldsymbol{\mu}})$. We arrive at the desired conclusion by a straightforward application of Corollaries 4.16, 4.17, Lemmas 4.18, 4.19 and 4.20 .

REMARK 4.21. The calculations for all the homogeneous operators constructed in [15] are not very different. However, we have not succeeded in completely answering the question raised in $[\mathbf{9}$, p. 39] using these calculations. Indeed, for generic bundles associated with the entire class of operators from [15], we have shown that the simultaneous unitary equivalence class of the curvature at 0 along with the covariant derivative of curvature at 0 of order $(0,1)$ is a complete set of unitary invariants for these operators.

\section{References}

1. J. Arazy and G. Zhang, 'Homogeneous multiplication operators on bounded symmetric domains', J. Funct. Anal. 202 (2003) 44-66.

2. N. Aronszajn, 'Theory of reproducing kernels', Trans. Amer.Math. Soc. 68 (1950) 337-404.

3. B. BAGCHI and G. Misra, 'Homogeneous tuples of multiplication operators on twisted Bergman spaces', J. Funct. Anal. 136 (1996) 171-213.

4. B. BAGCHI and G. Misra, 'Homogeneous operators and the projective representations of the Möbius group: a survey', Proc. Indian Acad. Sci. Math. Sci 111 (2001) 415-437.

5. B. Bagchi and G. Misra, 'The homogeneous shifts', J. Funct. Anal. 204 (2003) 293-319.

6. I. BiswAS and G. MiSRA, 'S $\widetilde{\mathrm{L}(2, \mathbb{R})}$-homogeneous vector bundles', Int. J. Math. 19 (2008) 1-19.

7. M. J. Cowen and R. G. Douglas, 'Complex geometry and operator theory', Acta Math. 141 (1978) $187-261$.

8. M. J. Cowen and R. G. Douglas, 'On operators possessing an open set of eigenvalues', Memorial Conf. for Féjer-Riesz, Colloq. Math. Soc. J. Bolyai, Budapest, 1980, 323-341.

9. M. J. Cowen and R. G. Douglas, 'Equivalence of connections', Adv. Math. 56 (1985) 39-91.

10. R. E. Curto and N. Salinas, 'Generalized Bergman kernels and the Cowen-Douglas theory', Amer. J. Math. 106 (1984) 447-488.

11. R. G. Douglas and G. Misra, 'Equivalence of quotient Hilbert modules-II', Trans. Amer. Math. Soc. 360 (2008) 2229-2264.

12. R. G. Douglas, G. Misra and C. Varughese, 'On quotient modules-the case of arbitrary multiplicity', J. Funct. Anal. 174 (2000) 364-398.

13. H. P. Jakobsen, 'Tensor products, reproducing kernels, and power series', J. Funct. Anal. 31 (1979) 293-305.

14. A. Korányi and G. Misra, 'New construction of some homogeneous operators', C. R. Acad. Sci. Paris, Ser. I 342 (2006) 933-936.

15. A. KorÁnyi and G. MisRa, 'Homogeneous operators on Hilbert spaces of holomorphic functions', J. Funct. Anal. 254 (2008) 2419-2436.

16. G. Misra and S. Shyam Roy, On Irreducibility of a class of homogeneous operators, Operator Theory: Advances and Applications (Birkhauser, Basel, 2007) 165-198.

17. L. Peng and G. Zhang, 'Tensor products of holomorphic representations and bilinear differential operators', J. Funct. Anal. 210 (2004) 171-192. 
18. R. O. Wells, 'Differential analysis on complex manifolds' (Springer, New York, 1973).

19. D. R. Wilkins, 'Homogeneous vector bundles and Cowen-Douglas operators', Int. J. Math. 4 (1993) 503-520.

Gadadhar Misra

Indian Institute of Science

Bangalore 560012

India

gm@math.iisc.ernet.in
Subrata Shyam Roy

Indian Statistical Institute

R. V. College Post

Bangalore 560059

India

ssroy@isibang.ac.in 